\title{
THE OPTICAL PROPERTIES OF SOME TRANSITION METALS
}

\author{
Ph.D. Thes is Submitted to lowa State University \\ February, 1973
}

\section{J. H. Weaver}

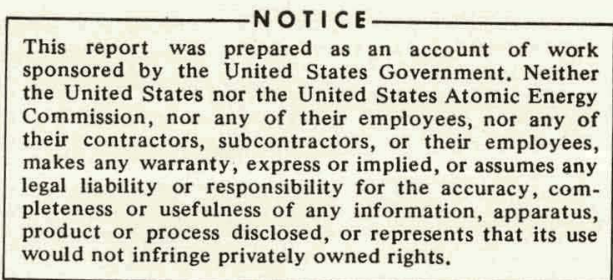

would not infringe privately owned rights.

Ames Laboratory, USAEC

lowa State University

Ames, lowa 50010

Date of Manuscript: February, 1973 


\section{DISCLAIMER}

This report was prepared as an account of work sponsored by an agency of the United States Government. Neither the United States Government nor any agency Thereof, nor any of their employees, makes any warranty, express or implied, or assumes any legal liability or responsibility for the accuracy, completeness, or usefulness of any information, apparatus, product, or process disclosed, or represents that its use would not infringe privately owned rights. Reference herein to any specific commercial product, process, or service by trade name, trademark, manufacturer, or otherwise does not necessarily constitute or imply its endorsement, recommendation, or favoring by the United States Government or any agency thereof. The views and opinions of authors expressed herein do not necessarily state or reflect those of the United States Government or any agency thereof. 


\section{DISCLAIMER}

Portions of this document may be illegible in electronic image products. Images are produced from the best available original document. 


This report was prepared as an account of work
sponsored by the United States Government. Neither
the United States nor the United States Atomic
Energy Commission, nor any of their employees, nor
any of their contractors, subcontractors, or their
employees, makes any warranty, express or implied,
or assumes any legal liability or responsibility for the
accuracy, completeness or usefulness of any
information, apparatus, product or process disclosed,
or represents that its use would not infringe privately
owned rights.

Available from: National Technical Information Service Department A

Springfield, VA 22151

Price: Microfiche $\$ 0.95$ 


\title{
The optical properties of some transition metals
}

by

John Herbert Weaver

\author{
A Dissertation Submitted to the \\ Graduate Faculty In Partial Fulfillment of \\ The Requirements for the Degree of \\ DOCTOR OF PHILOSOPHY \\ Department: Physics \\ Major: Solld State Physics
}

Approved:

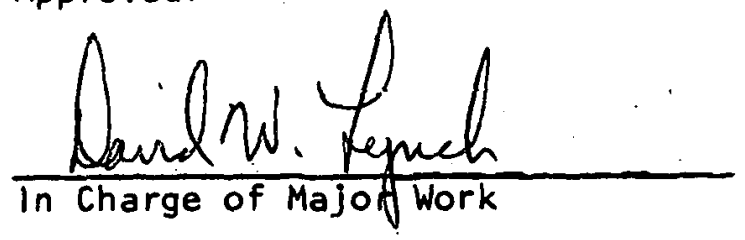

RG Barnes

For the Major Department

For the Erfduate college

lowa State University
Ames, lowa

1973 


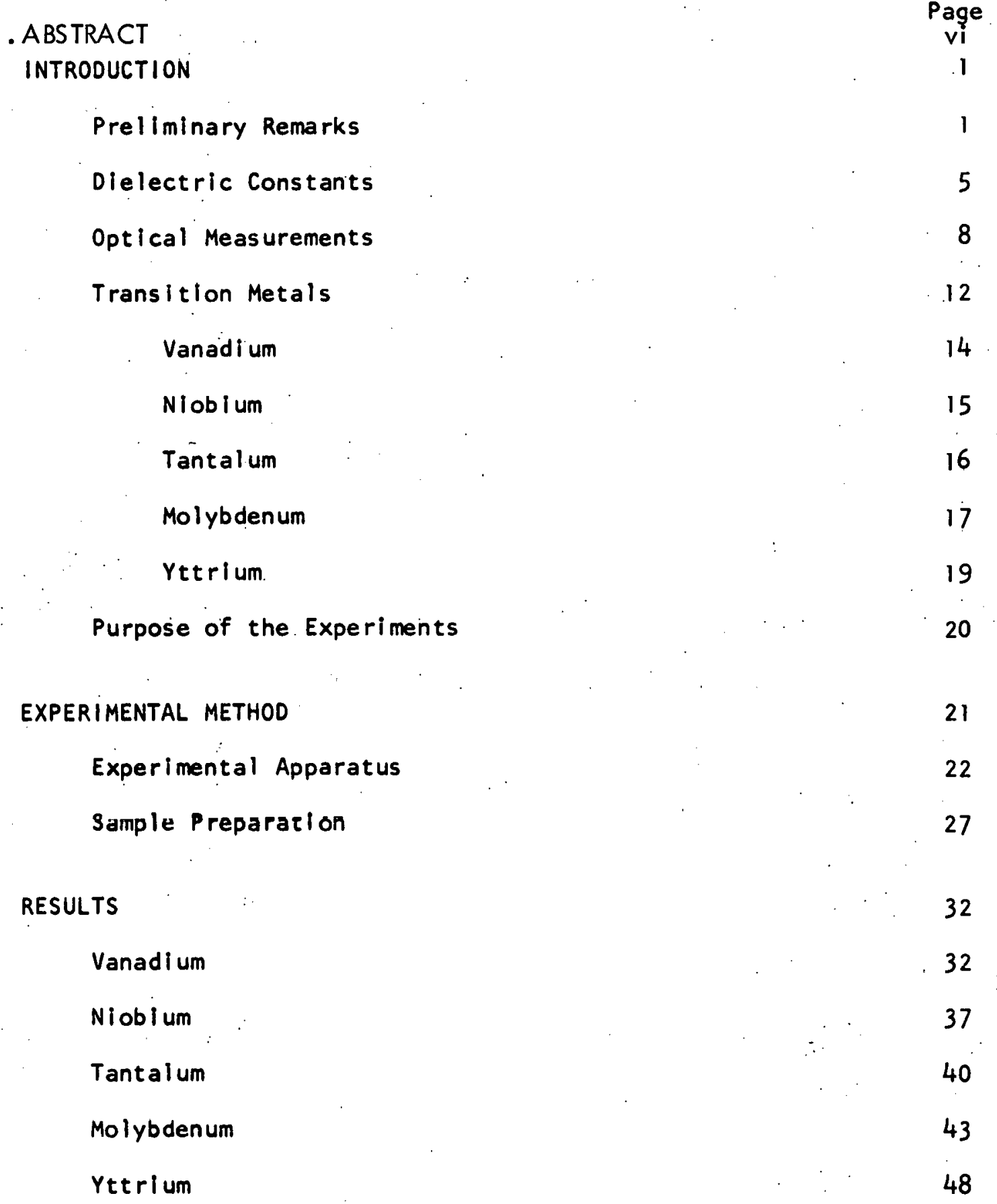


Page

DISCUSSION

50

Vanadi um

53

Niobium

57

Tantaium

59

Molybdenum

.62

Yttrium

64

CONCLUSIONS

67

BI BL I OGRAPHY

69

ACKNOWLEDGMENTS

74 
The optical properties of some

transition metals

John Herbert Weaver

Under the supervision of David W. Lynch

From the Department of Physics

lowa State University

The absorptivity of the bcc transition metals V, Nb, Ta and Mo and hcp $Y$ was measured calorimetrically at near-normal incidence in the energy range 0.1 to $4.88 \mathrm{eV}$. Unpolarized radiation was used for the bcc metals; for $Y$ the radiation was polarized to obtain the spectra with $\vec{E} \| \hat{c}$ and $\vec{E} \perp \vec{c}$. For the bcc metals, the low energy region was shown to be free of structure. Interband absorption was found to be large even at $0.15 \mathrm{eV}$ and was thought to persist to zero energy, although it did not contribute structure below the first strong absorption peak. For $Y$, low energy interband structure has been observed only for $\vec{E} \| \cdot \hat{c}$ at about $0.45 \mathrm{eV}$. The peak of the first strong absorption occul's al $1.35 \mathrm{eV}(\mathrm{V})$, about $1.8 \mathrm{eV}$ (Nb), $2.15 \mathrm{eV}$ ( $\mathrm{Ta}$ ) and $1.5 \mathrm{eV}$ (Mo). For the Vanadium serles, this has been identified as due to transitions like $\Sigma_{1}-\Sigma_{1}$; for Mo, the structure is probably due to transitions along $\Delta$. Structure which occurs in $\mathrm{Nb}$ and Ta at 5.1 and 5.3 eV, respectively, has been discussed in terms of the flat band extending along $P(D) N$. It is suspected that $V$ will also possess such a feature, but it occurs outside the range of the data presented here. Comparisons are made with existing optical data on these metals and 
the band structures are discussed where possible. For $V$, the shortcomings of the existing bands are pointed out, based on the optical data. The $Y$ results are interpreted based on the bands of Sc. 
INTRODUCTION

Preliminary Remarks

In recent years, the development of the technology involved in the purifying and growing of single crystals of materials has been paralleled by advances in the field of computer technology. With high purity crystals available, it has been possible to probe and map the topology of the Fermi surface using such highly sophisticated techniques as those of the de Haas-van Alphen (dHvA) effect, radio frequency size effect (RFSE), and cyclotron resonance. As experimental information has become available, and as the size of computers has grown, calculations of the band structure of metals have become feasible. Since it has been shown that a knowledge of the optical properties is of considerable assistance in discussing the electronic properties of materials, an increased interest in these properties has developed. The present study of certain transition metals was undertaken to investigate the optical properties over the wide energy range 0.1 to $35 \mathrm{eV}$; the region extending from 0.1 to about 4.9 eV will be discussed in this work. These data are viewed. as significant contributions to the store of experimental information available for the bcc transition metals V, Nb, Ta, and Mo and hcp Y. It is anticipated that they will be of assistance to those seeking to understand the transition metals and calculate their energy bands. Most of the reliable information about the optical properties of metals has come since the mid 1950's. Prior to that time, the available samples were of relatively low purity and were not handled properly (this will be discussed in detail later); further, it was not possible to 
interpret the results since fairly sophisticated band structures simply did not exist. It was not until 1962, for example, that the bands of Cu were calculated by Segall (1). Following these calculations, Ehrenreich and Philipp (2) investigated the optical properties of AI, Au and $\mathrm{Cu}$ by measuring the near-normal incidence reflectivity. They were able to formulate a reasonable picture of those transitions contributing to structure in the optical spectra. Subsequently the bands of a great many of the elements have been determined with varying degrees of success, and a wealth of information has been forthcoming. Many of the simple metals as well as the noble metals are now rather well understood. The understanding of the transition metals has been slower in coming, due both to a lack of experimental data and the difficulty in calculating the energy bands.

Probably the most successful and widely applied method for calculating the bands of the transition metals has been the Augmented Plane Wave (APW) method $(3,4)$. As is well known, a spherically symmetric 'muffin-tin' potential is assumed around each atomic site extending to some radius $R$, outside of which the potential is taken to be a constant. Suffice it to say that these potentials can be determined although it should be noted that there is considerable disagreement concerning, and current interest in, their formulation. The wave equation is then solved exactly within the spliere and in the Interstitial region subject to the boundary cundiliuns Invulved at $R$. The results of numerous calculations have been satisfactory, as judged by their ability to explain the experimental results. 
The application of the Orthogonalized Plane Wave (OPW) method (4) is complicated by the very nature of the transition metals. Not only do d-like conduction states converge slowly in the OPW formalism but the outermost $s$ and $p$ core states are not completely localized, thus preventing the orthogonalization of the plane waves to the outer core lovels (j). A mudlfication of the OPW method has been introduced, however, by Deegan and Twose (5) (for Nb) in which the wave function is made up of plane waves, orthogonalized to only the inner core states, and functions similar to 45 and $4 p$ atomic states. These states represent the behavior of the $s$ and $p$ functions and are explicity orthogonalized to the inner core levels. The results were quite satisfactory, as compared to the APW calculations for Nb by Mattheiss (6).

The pseudopotential model has recently been applied to the transition metals $(7,8)$. Fong and Cohen (9) have been successful in fitting the energy bands of $\mathrm{Cu}$ and $\mathrm{Nb}$ with an empirical pseudopotential by constructing the pseudo wave function from the usual expansion of plane Waves as well as additional d wave functions which are not strictly eigenfunctions of the crystal Hamiltonian.

The application of the cellular method to the transition metals has had only moderate success. Altmann and Bradley $(10,11)$ have considered the hcp metals, but these results are in conflict with the APW results of Loucks (12) and Fleming and Loucks (13). The problem may be due in part to a forced matching of the wave functions in regions of the lattice where the potential is quite flat. 
A modified form of the Wigner-Seitz method (4) as applied by Hodges et al. (14) has had greater success. A renormalized atom approach (which Segall used when calculating the bands of $\mathrm{Cu}$ ) is used which is basically a renormalization of the wave functions of free atoms. The free atom wave functions are truncated at the cell radius and, to guarantee charge neutralley, are renormalized within the cell. The method has considerable advantages, not the least of which is a knowledge of the wave function involved; this is not the case for the APW method, for example, in which the potential is determined by superimposed charge densities. With the renormalized atom approach to determine the potentials involved, the bands can be calculated and valuable information can be obtained readily about the center of gravity of the $d$-bands as well as their width, and the position of the conduction band minimum.

A variety of other models has been used in the calculation of bands for the transition metals, not the least of which is the KKR or Green's function method (4). Likewise, considerable activity is now apparent in groups trying to determine precisely what is needed in the potential. Modifications of existing schemes exist in which, say, the d electrons are treated by a tight-binding method while the conduction electrons are treated by an OPW or pseudopotential method. However, a comprehensive review of band theory is not the purpose of this study. Suffice it to say that there is considerable current interest in the transition metals and it is hoped that the data presented here will be of value to the theorists involved. 


\section{Dielectric Constants}

In the course of optical studies, numerous approaches are taken and various measured quantities are obtained, but ultimately all approaches lead to a determination of the complex dielectric function, $\widetilde{\varepsilon}$, or, equivalently, the complex index of refraction, $\widetilde{N}$. These are termed the optical constants, although of course both are functions of energy. The measured quantity is often the normal-incidence reflectivity $R$. which is expressed in terms of the complex reflection amplitude $\widetilde{r}$ as

$$
\begin{aligned}
R & =|\widetilde{r}|^{2} \\
\text { where } \quad \tilde{r} & =\frac{\widetilde{E}_{r}}{\widetilde{E}_{i}}=r e^{i \theta}
\end{aligned}
$$

and $\widetilde{E}_{r}$ and $\widetilde{E}_{i}$ are the reflected and incident electric field vectors. $\theta$ is the phase change upon reflection. If $R$ and $\theta$ are known, the optical constants can be calculated by solving the equation $\widetilde{r}=(\widetilde{N}-1) /(\widetilde{N}+1)$ for $n$ and $k$ where $\widetilde{N}$ is the complex dielectric function. The result is

$$
n=\frac{1-R}{1+R-2 \sqrt{R} \cos \theta}
$$$$
\text { and } \quad k=\frac{2 \sqrt{R} \sin \theta}{1+R-2 \sqrt{R} \cos \theta} \text {. }
$$

Since $\widetilde{\varepsilon}=\widetilde{N}^{2}$,

$$
\begin{aligned}
& \varepsilon_{1}=n^{2}-k^{2} \\
& \varepsilon_{2}=2 n k,
\end{aligned}
$$

following the convention that $\widetilde{\varepsilon}=\varepsilon_{1}+i \varepsilon_{2}$ and $\widetilde{N}=n+i k$. 
Then the reflectivity can be written (normal incidence) as

$$
R=\frac{(n-1)^{2}+k^{2}}{(n+1)^{2}+k^{2}}
$$

Since the physical quantities of interest are most readily discussed in terms of $\widetilde{\varepsilon}$, the following discussion will deal with $\tilde{\varepsilon}$ rather than $\widetilde{N}$.

The reflectivity $R$ carries contributions from two separate mechanisms: interband and intraband absorption. Electrons which are excited by the field but remain within the same band give rise to intraband absorption, while electrons which gain enough energy to move vertically to a higher band give rise to interband absorption. If the electrons in a metal are treated like a free electron gas and interband effects are neglected; the absorptivity $(A=1-R)$ due to intraband effects can be written

$$
A=2 / \omega_{p} T \text {, }
$$

where $\tau$ is the relaxation time, $\omega_{p}$ is the plasma frequency and it is necessary that $\omega \ll \omega_{p}$ and $\omega_{T} \gg 1$. The resulting absorptivity is small and energy-independent. Interband absorption complicates this simple relation. Since interband effects persist to zero energy for the transition metals (the Fermi surface cuts the d-band), this expression is especially rough for those metals.

To relate the experimentally determined dielectric function to a calculated dielectric function which carries within it the microscopic characteristics of the solid, one assumes that the Hamiltonian can be written as

$$
H=H_{0}+\frac{e}{m c} \vec{p} \cdot \vec{A}_{0} e^{-i \omega t}
$$


where $H_{0}$ is the crystal Hamiltonian and $A_{0}$ in the second term is the magnitude of the vector potential. The random phase approximation is made (15) and only the first term in the expansion of $\vec{A}$ is kept. It can be shown that $(15,16)$

$$
\widetilde{\varepsilon}(\omega)=1-\frac{\omega_{p}^{2}}{\omega\left(\omega+i / \tau_{c}\right)}-\frac{e^{2}}{\pi^{2} m} \int d^{3} k \sum_{\ell \ell^{\prime}} \frac{F_{\ell^{\prime}}(\vec{k}) F_{\ell^{\prime}}^{\mu^{\prime}}}{\left[\left(\omega+i / \tau_{\ell \ell^{\prime}}\right)^{2}-\omega_{\ell \ell^{\prime}}{ }^{2}\right]},
$$

where $h w_{\ell \ell^{\prime}}=E_{\ell}(\vec{k})-E_{\ell}(\vec{k}) . \quad E_{\ell}(\vec{k})$ is the energy of an electron in band $\ell$ with wave vector $\vec{k}, F_{l}(\vec{k})$ is the Fermi distribution and $F_{\ell l}^{\mu}$ is termed the oscillator strength

$$
F_{\ell l^{\prime}}^{u}=\frac{2}{\hbar \omega_{l \ell^{\prime m}}}|<\ell \vec{k}| p^{\mu}\left|\ell^{\prime} k>\right|^{2} \text {. }
$$

The state characterizes the electron in a state $\vec{k}$ in the band $l$ with $p^{\mu}$ being the component of momentum in the direction of polarization of the field. Finally, $\tau_{c}$ and $\tau$ characterize conduction band states and phenomenological lifetime broadening effects for interband transitions, respectively.

The first term of Equation 10 can be recognized as due to intraband or free carrier absorption and the second term involves interband effects, two bands being separated by the energy $\hbar w_{U L^{\prime}}$. Measurements of the reflectivity, and thus determination of the dielectric constants, thus provide information about the wave functions and electronic properties of the solid. It is useful to define a quantity termed the joint density of states (JDOS) as

$$
J_{U^{\prime}}(\omega)=\frac{1}{8_{\pi}^{3}} \int \frac{d S}{\nabla_{k}\left(E_{\ell}-E_{\ell^{\prime}}\right)} \mid E_{\ell}-E_{\ell^{\prime}}=\text { Aw }
$$


which is contained in $\epsilon_{2}$. When the JDOS is large, structure can be expected in $\varepsilon_{2}$.

While qualitative information can be gained from a knowledge of the energy bands themselves, more quantitative comparisons between experimental information and theory comes from a direct comparison of the measured $\varepsilon_{2}$ and an $\varepsilon_{2}$ calculated from Equation 10. Unfortunately, such calculations have rarely been performed; Kasowski (17) has done such calculation for $\mathrm{Cd}(18)$ and $\mathrm{Zn}$ (19) and the experimental results were in reasonably good agreement.

\section{Optical Measurements}

As shown above, optical measurements are concerned with determining the dielectric function. Historically, the most common approach has been to measure the reflectivity, $R$, or the absorptivity, $A(A=1-R)$. This can be done by measuring the incident beam and the reflected beam, or as is the case for the present study, the absorbed and reflected, beams. The advantages of the approach uzed here will be dlscussed in detail later.

It is well known that the real and imaginary parts of the response function which relates the linear response of a system to a generalized force ore related by the so-called dispersion relations $(20,21)$. These are statements of linearity and causality, i.e. the response to a force cannot precede the force, and must be directly proportional to the force. The dielectric function is such a response function, relating the electric field to the displacement field. If, then, the real part of $\tilde{\varepsilon}$ is known, 
the imaginary part can be determined. An expression which relates the two parts is generally termed a Kramers-Kronig integral. A KramersKronig (KK) integral can also be formulated that relates the reflectiv$i t y, R$, to the change of phase of the reflected beam relative to the incident beam, $\theta(20,21)$. A common form for the integral is

$$
\theta(E)=\frac{\omega}{\pi} P \int_{0}^{\infty} \frac{\ln R\left(\omega^{\prime}\right)}{\omega^{2}-\omega^{2} 2} d \omega^{\prime}
$$

where the integral extends from zero to infinite frequency, and the principal part is to be taken at the singularity. If the reflectivity is known over an infinite range of energies, the phase angle can be determined and the optical constants can be calculated. This points up the difficulty with reflectance measurements which is independent of the precision of the measurements themselves: the reflectivity is never known over an intinite range. Fortunately, the major contribution to the integral comes from the region near the singularity and it is customary to truncate the integral at sufficiently high and low energy. The meaning of 'sufficiently' is determined from the actual form of the numerical calculations.

When the reflectivity is measured, the energy range is restricted. To determine $\theta$, the range of energy is extended by using the results of other measurements at higher and lower energy, if such data exist, and extrapolating beyond the range of information. Clearly, this has its disadvantages. It assumes that 'patched' data are accurate and that there is good agreement in the region of overlap. Naturally, the resulting dielectric function has errors built into it by the KK analysis, 
even though the reflectivity has been measured very precisely in the range of interest. It is common to find disagreement in the magnitudes of $\widetilde{\varepsilon}$ although the basic shape of the structure observed is less affected by extrapolations.

Alternative approaches exist to the measurement of the reflectivity at a single angle of incidence. The determination of $R$ at multiple angles, one of which is large, allows the use of the Fresnel equations in the calculation $\tilde{\varepsilon}$.

Ellipsometry $(22,23)$ is another technique which avoids the KK difficulties. While the early data tend to be unreliable, more recent results are quite satisfactory. Instead of measuring the reflectivity, the amplitude ratio $\left|\tilde{r}_{p} \tilde{r}_{s}\right|$ is measured as well as the difference in the s- and p-phase shift upon reflection. Polarizers are used to adjust the components of the electric field vector $\vec{E}$ that are parallel (p) or perpendicular (s) to the plane of incidence. While the technique has its advantages, it suffers from a serious handicap: the phase shifts are strongly dependent on the purity of the surface. An oxide or contaminating layer will affect ellipsometric measurements to a far greater degree than near-normal incidence reflectivity measurements.

One of the more severe problems of optical measurements is related to the preparation of the sample surface. Different approaches require different degrees of flatness. In reflectivity measurements, a wavy surface scatters light and a portion may be lost to the detector; for ellipsometry this is less critical since light reaching the detector carries the correct information about the phases and the ratio of the 
reflectances. Errors introduced by the presence of an oxide are also common; in the case of near-normal incidence reflectivity, if the thickness and optical constants of the oxide are known, the oxide can be taken into account, but this information is generally not known. The rate of growth of the oxide, the stoichiometry and the optical constants of the oxlde are rarely known reliably. While for Al the oxide is known to be $\mathrm{Al}_{2} \mathrm{O}_{3}$ and have a stable thickness of $29 \AA$, the properties of the oxide of $Y$ are quite unknown. Fortunately, in many cases the oxides are transparent in the infrared and no corrections need be considered. To some extent the problems of an oxide can be avoided by cleaving single crystais in ultrahigh vacuum (uhv) and performing the measurements in situ. This is possible only for a limited number of metals and surface orientations. A more common approach which avoids the difficulties of polishing the crystal and oxides is to evaporate films in uhv and ayain perform the measurements in situ. This is not without serious drawbacks. Even if it is assumed that the characteristics of a film are identical to' those of a bulk crystal, the class of materials studied as films is limited to the cubic materials. It is difficult or impossible to evaporate films with all crystal orientations; for example, films of $\mathrm{Zn}$ preferentially display the basal plane. To measure the anisotropy of the basal and $\hat{c}$ plane is not possible. (This is done for crystals with polarized light with the electric field vector parallel or perpendicular to the $\hat{c}$-axis:)

In the preparation of bulk crystals, care is taken to polish them with sultable abrasives to obtain flat surfaces. For some materials 
this is a serious problem, but in any case the act of polishing has severely distorted the upper layers of the crystal. The extent of damage must be determined for each material and is determined to some extent by the polishing technique; it has been shown that for $\mathrm{Ni}$ a work-damaged layer extends as deeply as $200 \mu \mathrm{m}(24,25)$ into the surface. The need for the removal of this layer appears to be obvious, but a great deal of data exist in the literature which are, at best, representative of a badly distorted crystal. The damaged layer can generally be removed by chemical etches or electropolishes; the regime for which must be determined for the metal in question. While etches often leave the surface roughened, the electropolishing generally leaves a surface with a high degree of flatness and free from damage.

\section{Transition Metals}

linveslyarlons of the optical properties of the transition metals have been limited to a few select metals and generaily cover only a limited energy range. While the optiral measuroments of Llie noble and simple metals are quite advanced and the properties quite well understood, the transition metals have been left far behind. The problem of obtaining high purity single crystals still exists; practically all of the world's. Hf contains at least $2 \% \mathrm{Zr}(26)$ and single crystals of $\mathrm{Zr}$ are very rare. The existing, reasonably reliable studies will be revlewed for each metal separately in the following section and in the Discussion. For convenience, Figure 1 shows the location of the metals in the periodic chart and gives the outer electron configuration as well as the crystalline structure. 


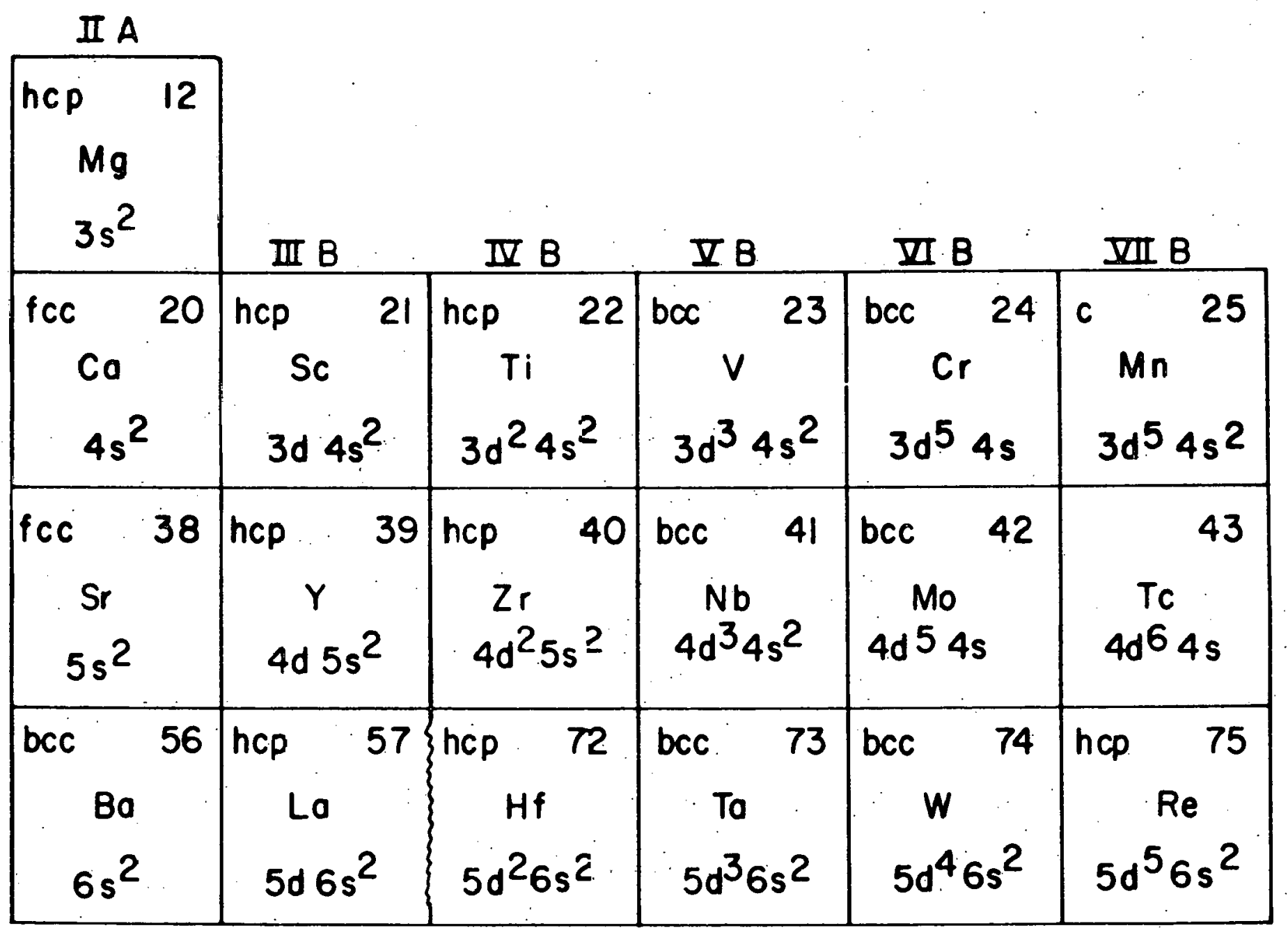

Figure 1. A portion of the periodic table showing the positions, electronic configurations, and crystal structures of the metals of interest. 


\section{Vanadium}

Most of the experimental studies of $V$ and its Fermi surface have come within the last 4 years. The paucity of experimental information prior to then did not warrant a complete band calculation, but recently a variety of theoretical studies have appeared.

The optical properties of $V$ have been considered by Bolotin et al. (27) who measured the optical constants $n$ and $k$ ellipsometrically at room temperature between 2 and $9 \mu \mathrm{m}$. Vilesov et al. (28) presented a reflectivity spectrum between about 5 and $15 \mathrm{eV}$, but their results bear little resemblance to the more recent work of Seignac and Robin (29). Seignac and Robin determined the optical properties as based on their reflectivity measurements of thin $V$ films between 3 and $50 \mathrm{eV}$ (250 to 4000 \&). Optical absorption measurements have also been reported between 30 and $300 \mathrm{eV}$ by Sonntag et al. (30) and show core transitions in the vicinity of $50 \mathrm{eV}$. Photoemission studies by Eastman. (31) have provided information about the placement of the d-bands of $V$. The data from 0.15 to $4.88 \mathrm{eV}$ presented here thus represent the only reliable data in the visible and infrared; a certain scepticism exists concerning the data of Seignac and Robin and plans exist for improved high energy measurements.

The energy bands of $V$ have been calculated with varying degrees of completeness. Mattheiss (32) displayed $E(\vec{k})$ along the line $\Gamma(\Delta) H$ and Snnw and Waber (33) considered $V$ in conjunction with the $3 d$ series of transition metals. Neither work provided a detailed band scheme although Mattheiss did subsequently discuss the $V$ group briefly in terms 
of the bands and Fermi surface of W (34). More recently, APW calculations by Anderson et al. (35) showed the bands along $\mathrm{N}-\Gamma-\mathrm{H}$ for two different values of the exchange potential coefficient and two values of the lattice spacing. The same group (36) subsequently completed a self-consistent APW calculation giving the bands at points of high symmetry. Yasui et al. (37) also self-consistently evaluated the bands with two different exchange term coefficients, treating the d electrons by a tight-binding method and the conduction bands by an OPW method. Hodges (38) has calculated the bands, but the results have not been fitted to experimental results as of this date. The various bands will be compared and discussed in conjunction with the optical data later.

The Fermi surface of $V$ has been studied using the technique of magnetoresistance by two groups. Alekseevski i and Egorov (39) reported practically no anisotropy while Nelson et al. (40), working with samples of higher purity, reported anisotropy. Phillips (41) has performed de Haas-van Alphen measurements. Finally, thermomodulation experiments of Weaver et al. (42) have provided information about the bands near the Fermi level.

\section{Niobium}

Niobium lias been scudied more often, if not more reliably, than $v$. The most recent optical work with Nb was that of Golovashkin et al. (43). They employed an ellipsometric technique in the 0.4 to $10 \mathrm{um}$ range and made measurements at 293, 78 and $4.2 \mathrm{~K}$. Considerable attention was given to sample preparation and it was reported that surface damage extended a 
depth of $50 \mu \mathrm{m}$ into the surface. An electropolish was used to remove this worked layer. The earlier work by Vilesov et al. (28) also recognized the need for chemical polishing. Their reported reflectivity spectrum extended from 3.1 to $14.3 \mathrm{eV}$ and appears to be more reliable than their $V$ spectrum, at least over part of the energy range. other early work (44) was carried out with samples that were merely mechanically polished and is less representative of an unstrained Nb crystal lattice.

A variety of experimental information has recently been obtained about the Fermi surface of $\mathrm{Nb}$. Galvometric (45) magnetothermal oscillation (46), and de Haas-van Alphen $(46,47,48)$ measurements have been performed. From the theoretical point of view, the electronic structure was being considered by Mattheiss (6) (APW) and earlier by Deegan and Twose (5) (nonorthogonalized OPW as discussed above). Fong and Cohen (9) have also been able to use an empirical pseudopotential to determine the band structure. The self consistent APW calculations of Anderson et al. (49) were also in agreement with the results of Mattheiss.

\section{Tantalum}

The existing optical experiments on Ta are limited to the ultraviolet and visible regions of the spectrum. Juenker and co-workers $(50,51)$ determined the reflectivity of high-temperature cleaned surfaces of rolled Ta metal. The agreement with our data is rather good in the. visible; their uv data are in basically good agreement insofar as shape 
is concerned but the magnitude of their reflectivity is high, presumably due to scattered light.

The electronic structure of Ta was considered by Mattheiss (6) in the same paper as $\mathrm{Nb}$. $\mathrm{Nb}$ was considered in the nonrelativistic limit, but for Ta relativistic effects were considered. A considerable similarity was noted for the Fermi surfaces and bands near the Fermi level. This has been experimentally supported by recent dHvA $(46,47,48)$, magnetothermal oscillation (48) and galvanometric (45) measurements. Our optical data do demonstrate that there is similarity, but not to the extent suggested by the similarity in the Fermi surfaces. It will be seen that $T a$ has the simpler optical spectrum and is easier to interpret based on the bands of Mattheiss.

\section{Molybdenum}

Optical investigations of Mo have been carried out earlier by other groups. Kirillova et al. (52) investigated the range 0.06 to $4.9 \mathrm{eV}$ using an ariented singlo orystal and all elllpsometic technique at room temperature. In an earlier paper, Kirillova and others (53) reported a reflectivity spectrum for Mo extending to $15 \mathrm{eV}$. The results were in reasonably good agreement with those reported by Juenker and co-workers $(50,51)$ who investigated the visible and vacuum ultraviolet to about $24 \mathrm{eV}$. While the agreement between these results and ours is rather good, other groups have found vastly different $R$ spectra. Kapitsa et al. (54) measured $R$ from 1.4 to $11 \mathrm{eV}$, but their values were much lower in magnitude than those mentioned above; the only common characteristic is 
a minimum in $\mathrm{R}$ at about $3.5 \mathrm{eV}$. Kress and Lapeyre (55) also reported a reflectivity spectrum from 0.5 to $14 \mathrm{eV}$ but it is unlike any of the others between 4.5 and $9 \mathrm{eV}$. The present data thus support those of Kirillova et al. (52) above about $1 \mathrm{eV}$ and agree basically with Juenker et al. in the common region. Below about $\mathrm{l}$ eV, our absorptivity values are lower than any previously reported, suggesting a better sample and surface preparation. While it was suspected that a low energy interband transition might be found in Mo, results from an experiment by Lind seem to indicate that the transition lies at energies less than $0.06 \mathrm{eV}$, in agreement with the results of Nomerovannaya et al. (56) for $W$.

Molybdenum has been considered in some detail both experimentally and theoretically. Numerous de Haas-van Alphen $(57,58,59)$, radio frequency size effect $(60,61)$, magnetoresistance $(62,63,64)$, anomalous skin effect (65), magnetoacoustic effect (66) and cyclotron resonance (67) measurements have contributed information about the Fermi surface of Mo. The first theoretical contribution was made by Lomer (68) who proposed a model for the Cr group based on the APW band calculations of Wood for Fe; Lomer (69) later modified the model and applied it to Mo along. APW calculations for $\mathrm{Cr}$, Mo and $\mathrm{W}$ by Loucks (70) have provided a picture of the Fermi surface and are in qualitative agreement with the model of Lomer, but quantitatively the results are not reliable due to an error in the magnitude of the unit cell used in the calculations (71). It has been shown, moreover, that the band structures

\footnotetext{
${ }^{1}$ The reflectivity was measured at $300 \mathrm{~K}$ to $20 \mu \mathrm{m}$ but no structure was observed.
} 
of Mo and $W$ are quite similar if relativistic effects are ignored. Mattheiss (72) has calculated a reasonably detailed nonrelativistic band structure for $W$. Most recently, Iverson and Hodges (73) have applied a tight-binding interpolation scheme to renormalized atom calculations of the Mo bands (14). Spin-orbit effects have also been considered by Loucks (70) and Mattheiss (72) for $W$ and by Iverson and Hodges for Mo (73); the splitting of the bands along $\Gamma-H$ has produced a separation between the electron jack and the hole octahedron segments of the Fermi surfaces. The gap for Mo has been estimated to be about $2.5 \%$ of the $\Gamma \mathrm{H}$ dimension although there has been some disagreement between recent RFSE (61) and dHvA (57) results; the latter being favored. Bands along $\Gamma H$ which are cut by the Fermi level are known to be separated by about $i-2$ $\mathrm{eV}$ and low energy structure in the optical constants has been attributed to these transitions.

\section{Yttrium}

Experimental work with hop $Y$ is practically nonexistent, perhaps because single crystals of high purity have not been available. The transmission of semi-transparent films of $Y$ evaporated in high vacuum has been studied from 2000 to $8000 \AA$ by Petrakian et al. (74) but an oriented single crystal has never been studied.

The Ferml surface of $Y$ has been considered by Loucks (75) but there have been no band calculations per se. However, it has been pointed (75) out that $Y$ is very similar to some of the other hcp transition metals and the heavier $4 f$ rare earth metals. APW calculations have shown (76) that the density of states curves of $\operatorname{Re}(77), \mathrm{Gd}(78), Y \cdot(75)$, and Dy 
(79) are quite similar. One might further expect a resemblance of $Y$ to $\mathrm{Sc}$ (13). While a degree of similarity will exist, certain differences can, or course, be expected. The results obtained here will be interpreted in terms of the bands of Sc though it is clear that only qualitative comparisons can be made. Nonetheless, our data do tend to confirm the bands of Sc, which have not been subjected to the scrutiny of optical measurements.

\section{Purpose of the Experiments}

As indicated above, the recent interest in the transition metals has been stimulated both by improved calculational schemes and improved sample purity. While the optical properties of a metal are of considerable interest and assistance in the interpretation of the electronic properties, there have been no systematic and reliable optical measurements of the transition metals. Accordingly, this study was undertaken; when the data presented here are viewed in conjunction with concurrent vuv measurements, an understanding of tho behovior of Llie electrons in a solid can be gained. 
EXPERIMENTAL METHOD

A variety of experimental approaches can be taken to determine the optical spectra of a metal. One of the conceptually simplest and experimentally most straightforward involves the measurement of the absorptivity by a calorimetric method. The approach is as follows: monochromatic light is focused on a sample at near-normal incidence; a fraction of the incident radiation is absorbed and the remainder is reflected; the reflected beam is incident upon, and absorbed by, a nearlyperfectly absorbing surface. Since the sample and absorber are at near $4.2 \mathrm{~K}$, their heat capacities are small and both experience a temperature increase. By interrupting the light beam and reproducing the temperature rise with heaters attached to the two active elements, and by measuring the power dissipated by the heaters, it is possible to determine the traction of light absorbed by the sample, and thereby the absorptivity.

A clear advantage of calorimetry is that the measured quantity is the absorptivity rather than the roflectivity. III. Lhe Infrared, the reflectivity is large and at $0.1 \mathrm{eV}$ typically approaches 0.97 or more. To measure the reflectivity to an accuracy of $1 \%$ (relative) is then vastly inferior to measurement of the absorptivity to the same $1 \%$ accuracy. The result is that structure in the infrared can be more clearly delineated with calorimetry than by other techniques. (Improved modulation spectroscopy in the infrared will represent improvements over calorimetry, but very few such experiments have been performed to date.) As indicated above, ellipsometry is highly sensitive 
to surface contamination, and low temperature work is particularly sensitive to condensates. Near-normal reflectivity measurements are less sensitive to transparent gases, but care was taken to avoid these problems, as will be discussed subsequently.

\section{Experimental Apparatus}

The details of the calorimeter and the optics are indicated in Figure 2. A Leiss double prism monochromator provides the monochromatic light which is then directed down, the tube, through a radiation shield and focussed on the sample. The chamber itself is immersed in llquid hellum as shown. The details of the sample flange are shown in Figure 3. The light not absorbed by the sample is reflected and absorbed by the absorber. The radiation shields above the sample and the absorber are coated with the same Au-black as is the absorber itself and serve to eliminate problems from scattered light. They guarantee that only light incident upon the sample reaches the absorber, and conversely.

The temperature of the two elements is monitored hy $47 \mathrm{shm} \mathrm{Allon}$ Bradley resistors which have large temperature coefficients of resistance and make it possible to measure temperature changes of millidegrees. These thermometers and the $1000 \mathrm{ohm}$ IRC heater resistors (matched metal film precision resistors) are attached thermally to the elements.

The electrical path of the leads is not as shown in Figure 3 . Previously, a 12 pin stupakoff electrical feedthrough was soft-soldered to the copper insert which was hard-soldered to the flange itself. The leads were soldered to the pins and brought out through the helium bath. 


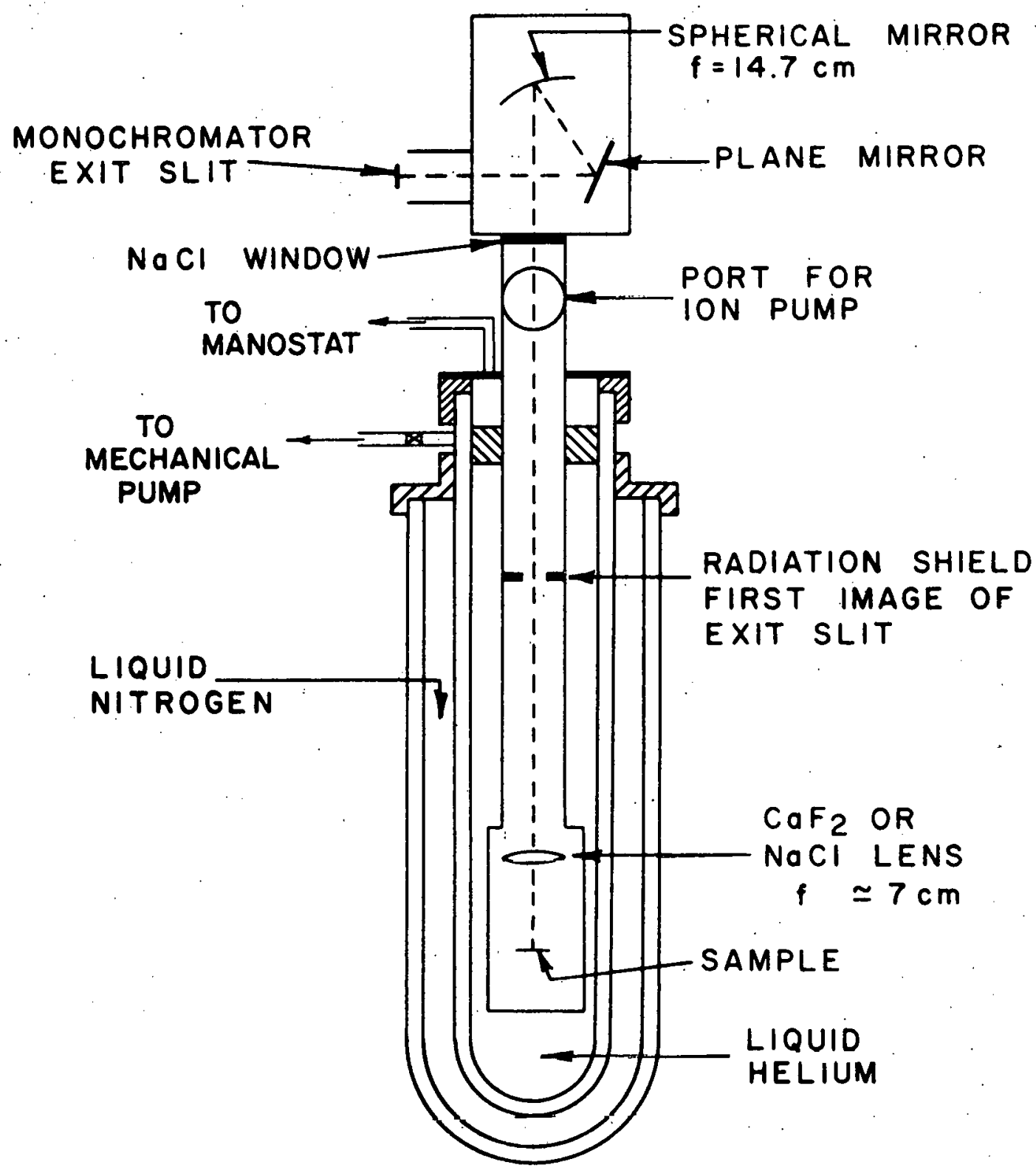

Figure 2. Overall schematic of the calorimeter showing the light path and dewar system 


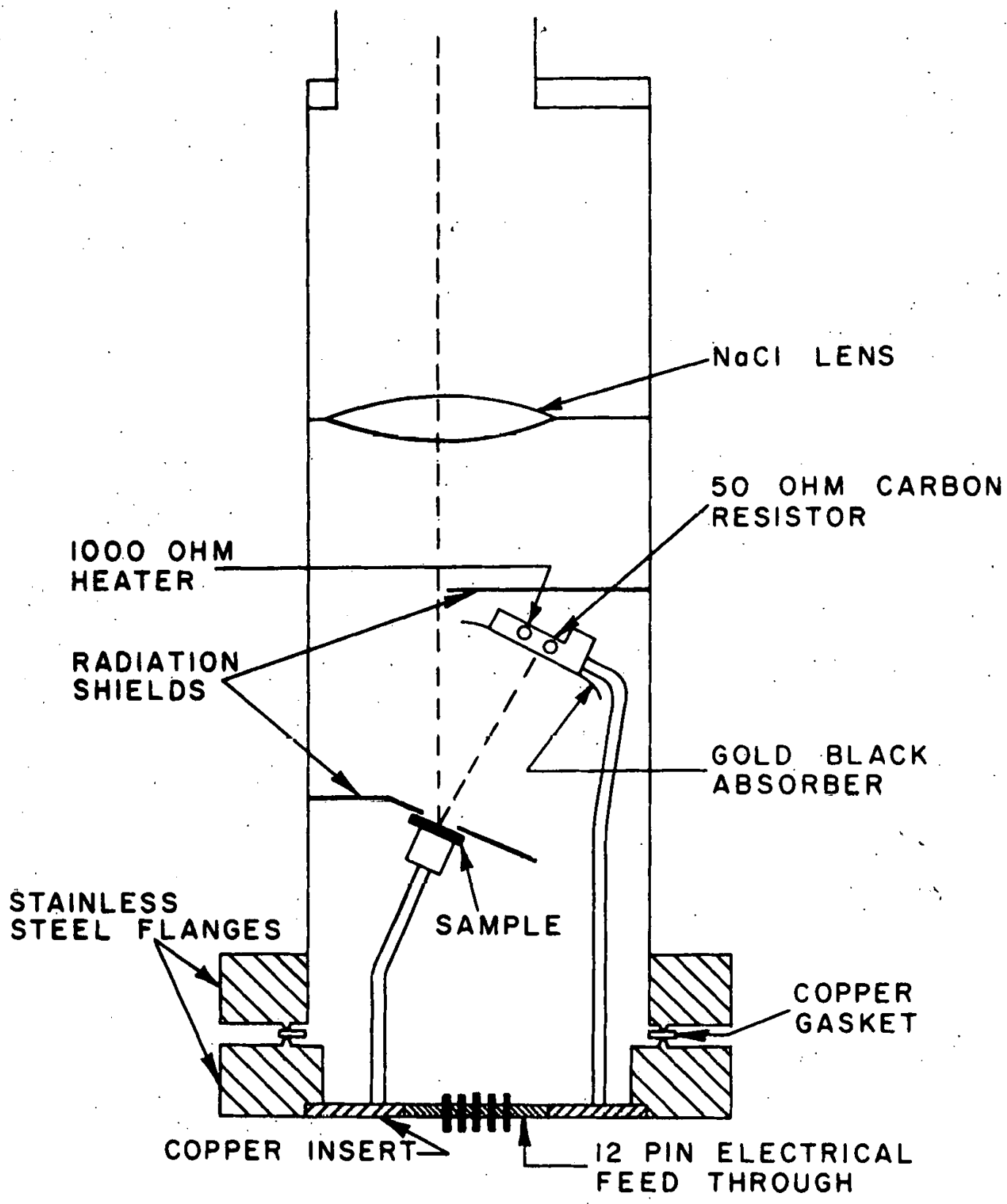

Figure 3. Detalled schematic of the sample flange and the optical path 
With thermal cycling the differential coefficients of expansion produced vacuum leaks with some regularity. To avoid the problem, the leads were passed through a stainless steel tube which was hard-soldered to the copper insert and bent appropriately to bring the leads out at room temperature. A Torr Seal feedthrough was made and served as a room temperature feedthrough. To eliminate a thermal short between the sample and the room temperature end of the wires, the $\mathrm{Cu}$ leads were thermally grounded at the flange. Cu clamps were attached to the flange and were tightened with the leads between them. Further isolation was guaranteed by constantan leads which lead directly to the sample and absorber resistors and were soldered to the grounded Cu wires. The only contact either element had with the bath was provided by $20 \mathrm{~cm}$ long. \#32 cu leads. These served as a heat leak and allowed the elements to rise above the bath temporaturo. Adjustimenl of the length and diameter of the heat leak allowed variation of the time constants involved, the time constant being determined by the thermal resistance of the lead and the thermal caparity of the absorber or sample. Further, the thermal isolation of the sample also guaranteed that no gases condensed on the sample as it cooled. Not only was the chamber pressure better than $\sim 10^{-5}$ Torr (room temperature), but during the cooling, the sample was the last part to cool. In fact, after the liquid helium was transferred and the calorimeter was immersed in the bath, it typically took $\sim 1 \mathrm{hr}$ before the sample cooled to $4.2 \mathrm{~K}$.

The procedure for cycling the calorimeter was as follows: the sample was attached to the Cu block, the shields were repositioned, the flange mated to the calorimeter base flange and the bolts were attached. 
As the bolts were secured, the chamber was flushed with high purity $\mathrm{N}_{2}$. This purging not only retarded oxidation of the sample, but also served to reduce the pump-out time, the amount of absorbed water vapor being minimized. The calorimeter was then immediately placed in the dewar, secured and evacuated. A forepump was used for roughing and a coldtrapped diffusion pump provided a vacuum of $\sim 10^{-5}$ Torr. Routinely $\sim 1$ hr elapsed before the ion pump was started and liquid nitrogen was transferred into the outer dewar. The calorimeter cooled in about 2 hrs to about $105 \mathrm{~K}$, a back pressure of $\sim 150 \mu \mathrm{m}$ of $\mathrm{N}_{2}$ serving as an exchange gas in the inner dewar vacuum chamber. Finally, liquid helium was transferred. It should be noted that the above procedure abbreviates that outlined by Bos $(80)$. With the above modifications, it was possible to take data within $\sim 4$ hrs of the time the sample was electropolished. Indeed, it was possible to completely cycle the calorimeter in 24 hrs, allowing 4-6 hrs for a complete energy scan of from 0.10 to $4.88 \mathrm{eV}$. The accuracy of measurements of the absorptivity is estimated to be better than $1 \%$ of the measured quantity. Thus, for a given run, the scatter of the data was within the thickness of lines drawn to display A (see Figure 4, for example). The reproducibility from run to run was also excellent and differences were generally attributed to the surface quality.

In the course of the studies, the Au-black on the shields and absorber was replaced as needed. The technique for producing highly absorbing Au-black was outlined by Bos ( 80$)$. The difficulty of getting the Au to wet the $W$ wire was overcome by initially pumping out the bell 
jar, then giving the W wire a current surge to quickly heat the element. This melted the Au and the Au wetted the wire. A more gradual increase in the temperature of the element usually resulted in the loss of much of the Au.

The procedure for taking data has been outlined by Bos ( 80 ) and remains largely unchanged. Likewise, the range of the various prisms and light sources, and the choice of polarizers remain as described. Similarly, the characteristics of the Au-black are as they were.

\section{Sample Preparation}

The samples of $\mathrm{V}, \mathrm{Nb}, \mathrm{Ta}$ and Mo were spark cut from buttons of electron beam melted metal obtained within the Ames Laboratory. The buttons were cut and etched and the samples were cut to take advantage of the largest crystallites. Typically, sample dimensions were $8 \times 12 \mathrm{~mm}$ with a thickness of $1 \mathrm{~mm}$. Most of the surface exposed to radiation was a single crystallite; the dimensions of the crystallites are given in Tahle.1. The samplos were chemically analyzed and the results are also shown in Table 1. A measure of the impurity concentration, the residual resistivity ratio ( $300 \mathrm{~K}^{/ \rho_{4}} 4.2 \mathrm{~K}$ ) was measured and is also given in Table 1. It is seen that the samples were of high purity. Indeed, our crystal of $V$ had a higher resistivity ratio than any reported in the literature, the sample of Nelson et al. (40) excepted.

The sample of $Y$ was treated differently. Since $Y$ has an hcp crystal structure, it was necessary to have a single crystal of known orientation. High purity starting material was obtained within the Ames Laboratory. 
Table 1. Sample characteristics

\begin{tabular}{|c|c|c|c|}
\hline Sample & $\begin{array}{c}\text { Characteristic } \\
\text { crystallite } \\
\text { dimension }\end{array}$ & $\begin{array}{l}\text { Residual } \\
\text { resistivity } \\
\text { ratio }\end{array}$ & $\begin{array}{l}\text { Chemical } \\
\text { analysis } \\
\text { (ppm) }\end{array}$ \\
\hline v & $5 \mathrm{~mm}$ & 200 & $\begin{array}{lc}0: & 50 \\
N: & 1 \\
\text { C: } & 29 \\
\text { Cr: } & -a\end{array}$ \\
\hline $\mathrm{Nb}$ & single & 100 & $\begin{array}{lc}0: & 9.6 \\
\mathrm{~N}: & 11 \\
\mathrm{C}: & 8 \\
\mathrm{Ta}: & <150^{\mathrm{b}} \\
\mathrm{Ti} & <100^{\mathrm{b}}\end{array}$ \\
\hline $\mathrm{Ta}$ & $2 \mathrm{~mm}$ & 21.7 & $\begin{array}{ll}0: & 70 \\
\mathrm{~N}: & 10 \\
\mathrm{C}: & 43 \\
\mathrm{Fe}: & -\mathrm{a} \\
\mathrm{Mg}: & \mathrm{e}\end{array}$ \\
\hline Mo & $\begin{array}{c}5 \mathrm{~mm} \\
\text { (bi-crystal) }\end{array}$ & 193 & $\begin{array}{lr}0: & 8 \\
\mathrm{~N}: & 1 \\
\mathrm{C}: & 22 \\
\mathrm{Fe}: & -\mathrm{a} \\
\mathrm{Ni}: & -\mathrm{a}\end{array}$ \\
\hline$Y^{d}$ & $\begin{array}{l}\text { single } \\
\text { (oriented) }\end{array}$ & & $\begin{array}{lr}0: & 227 \\
\text { N: } & 25 \\
\text { H: } & 11 \\
\text { C: } & 70 \\
\text { W: } & 40 \\
\text { NI: } & 40 \\
\text { Fe: } & 18 \\
\text { La: } & 13 \\
\text { Ce: } & 28 \\
\text { F: } & 8 \\
\text { Cl: } & 9 \\
\text { Ta: } & 5 \\
\text { Si: } & 4 \\
\text { Cu: } & 3 \\
\text { Tb: } & 3.2\end{array}$ \\
\hline
\end{tabular}

arace.

b Lowest level of sensitivity.

CFaint trace.

${ }^{d}$ Analysis of starting material; impurity less than 1 ppm not listed. 
It was formed into two cylinders of approximately $50 \times 12 \mathrm{~mm}$ which then were strain-annealed. The resulting cylinders were essentially single crystals, small crystallites being visible only at the ends. One cylinder was oriented using a back reflection Lave technique and single crystals with the $\hat{c}$-axis along one edge were cut for optical measurements.

After the crystals were cut to size, they were etched and polished using a final grade of $1 \mu \mathrm{m}$ alumina abrasive with alcohol or water as a carrier. The resulting surfaces were excellent. The samples were then electropolished ( $V$, Mo, Ta) or chemically cleaned (Nb) and annealed as shown in Table 2. The solutions used for electropolishing or cleaning are listed In Table 2. For annealing, the crystals.were sealed in Arfilled Ta capsules (low temperature annealing) or vacuum sealed in Ta capsules (high temperature annealing). After annealing, the samples were cleaned and re-electropolished chemically polished. Following the final electropolish, the samples were washed in alcohol, absolute (ethyl) alcohol and dried in a stream of high purity $\mathrm{N}_{2}$. They were then mounted as discussed above.

The crystals of $Y$ were more difficult to polish due to a tendency to pit. It was possible, with very careful polishing to a final grade of $0.05 \mu \mathrm{m}$ abrasive, to obtain a surface nearly free of pits. It should be noted that a sample with pits visible to the eye was also run and. the results were practically identical to those obtained with the flatter surface. This can be attributed to the large surface area of the absorber which collects all but a very small fraction of the light 
Table 2. Thermal and chemical treatment of the samples

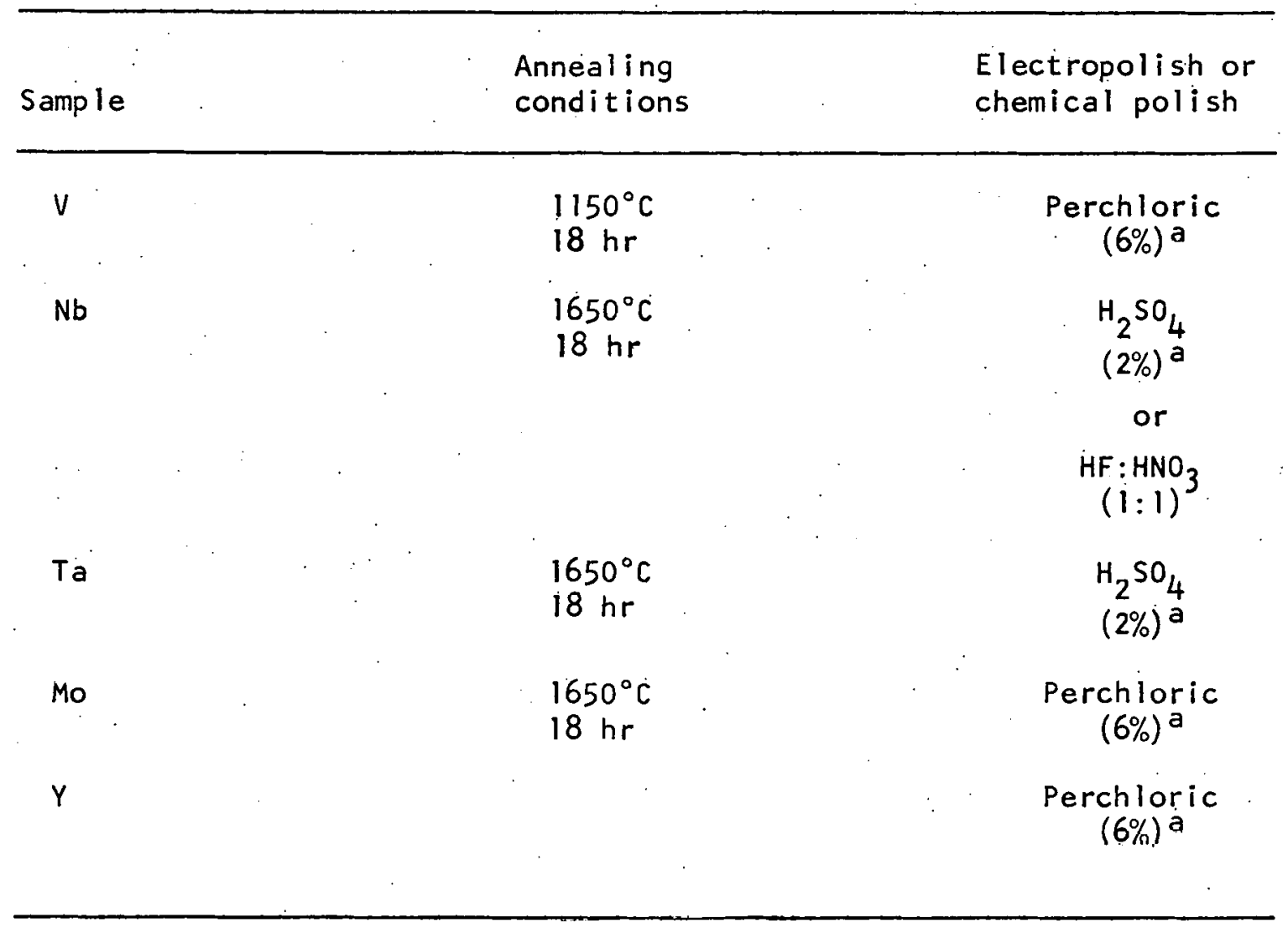

$a_{\%}$ by volume in methanol, cooled by solution of dry ice + acetnne. 
reflected, specularly or otherwise, from the sample. As usual the $Y$ crystals were electropolished. It was found that for $Y$ the surface had to be very thoroughly cleaned (ultrasonically with alcohol but not acetone) or the surface would discolor as it dried.

As discussed above, it has been shown that the surface damage introduced by mechanical polishing extends deep into the surface. For Nb; for example, Golovashkin et al. (43) demonstrated that as much as $50 \mu \mathrm{m}$ must be removed to get a strain-free surface. Accordingly, care was taken to remove a considerable amount of the surface prior to studying the sample. The exact amount of removed material is not known but the amount is estimated to be in excess of $50 \mu \mathrm{m}$. As a check of the strain on the surface, the samples were $x$-rayed. The surfaces showed none of the distorted $x$-ray spots characteristic of strain. 


\section{RESULTS}

Vanadium

The measured absorptivity of $V$ is shown in Figure 4 . No interband features are noted in the infrared below the first major absorption peak which occurs at $1.35 \mathrm{ev}$. The absorptivity rises smoothly from 0.0233 at $0.15 \mathrm{eV}$. A second peak in A occurs at about $2.15 \mathrm{eV}$ above which A displays a broad minimum before rising to the upper limit of our data.

A comparison of our infrared results for $V$ with those of Bolotin et al. (27) reveals that our A values are approximately half theirs, and their A rises approximately linearly with E. This will be discussed in more detail in a subsequent section. In the ultraviolet region of overlap (approximately 3.5 to $5 \mathrm{eV}$ ), the data of Seignac and Robin (29) are in reasonabiy good agreement.

In order to determine the optical constants, it is necessary to... Kramers-Kronig (KK) analyze the reflectivity to determine the phase angle. and thence to calculate $\widetilde{\epsilon}$, as discussed above. In the absence of high energy data obtained as part of this study, the results of seignac and Robin have been used. The complete reflectivity spectrum then consists of our data from 0.15 to $4.88 \mathrm{eV}$, a smooth interpolation from 4.88 to 5.5 eV joining our data to those of Seignac and Robin, and the latter's data to about $30 \mathrm{eV}$. Above $30 \mathrm{eV}$, the reflectivity was calculated from the absorption measurcments of Sunntag et al. (30) and extended to $100 \mathrm{eV}$ whore $R=0.0001$ and the integral was truncated. In the infrared beyond our range, smooth extrapolations were introduced to reduce $A$ to zero at zero energy. 


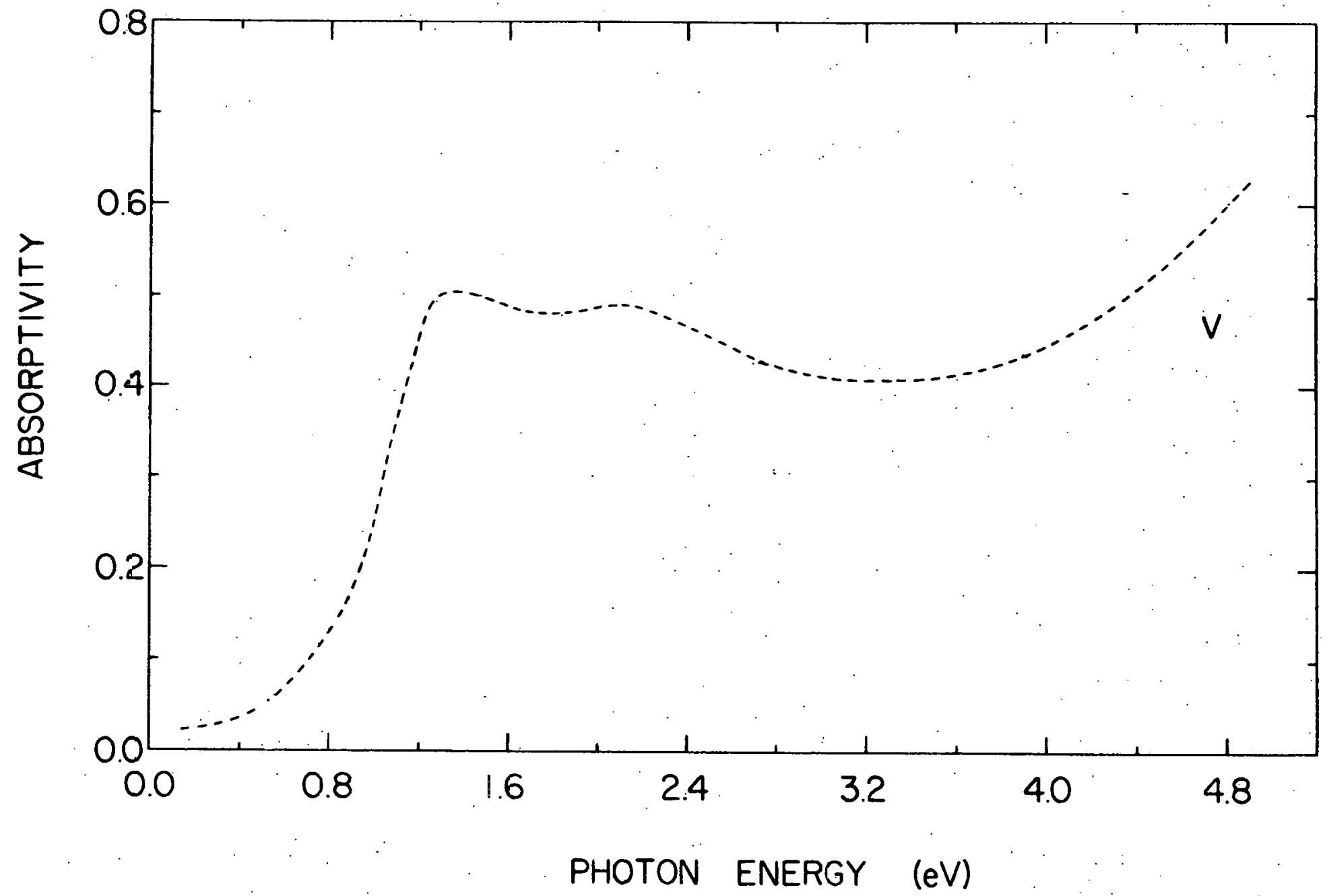

Fgiure 4. The absorptivity of $V$ from 0.15 to $4.88 \mathrm{eV}$ at $4.2 \mathrm{~K}$ and near-normal incidence 
The real and imaginary parts of the complex dielectric function are shown for $V$ in Figure 5. As discussed above, due to the nature of the KK analysis, some uncertainty in the magnitude of the constants exists, although the shapes of the curves are probably unaffected by the choice of high energy extrapolations. It is expected that after the measurement of $R$ to $35 \mathrm{AV}$, the constants will have yreater reliability insofar as their magni tudes are concerned.

Structure in $\epsilon_{2}$ is apparent at 1.65 and $2.45 \mathrm{eV}$ and $\epsilon_{1}$ is seen to cross the axis (just barely) at $1.5 \mathrm{eV}$. At lower energy, below the onset of strong interband structure, $\widetilde{\epsilon}$ basically displays the characteristics of free electron or free carrier absorption: $\epsilon_{2}$ is large and positive and drops rapidly toward zero; $\epsilon_{1}$ is large and negative and rises quickly toward zero.

The effective number of electrons per atom contributing to absorption processes below energy $E$ can be calculated from the oscillator strength sum rule of the form

$$
N_{e f f}(E)=\frac{2}{\pi} \frac{1}{\left(\hbar \omega_{p}\right)^{2}} \int_{0}^{E} \epsilon_{2}\left(E^{\prime}\right) E^{\prime} d E^{\prime},
$$

where $w_{p}$ is the plasma energy, $\omega_{p}{ }^{2}=\frac{4 \pi \mathrm{Ne}^{2}}{m}$, there being $N$ electrons of mass $m$ per unit volume. In $V$ where $N$ was 5 electrons per atom, the free electron plasma energy was calculated to be $22.3 \mathrm{eV}$. N $N_{\text {eff }}$ is shown in Figure 6, from which ic can be seen that 0.7 electrons per atom have cuntrlbured to the oscillator strength between 0.1 and $4.88 \mathrm{eV}$. since this is highly dependent upon the magnitudes of $\varepsilon_{2}$, only the shape of Neff should be relied upon. 


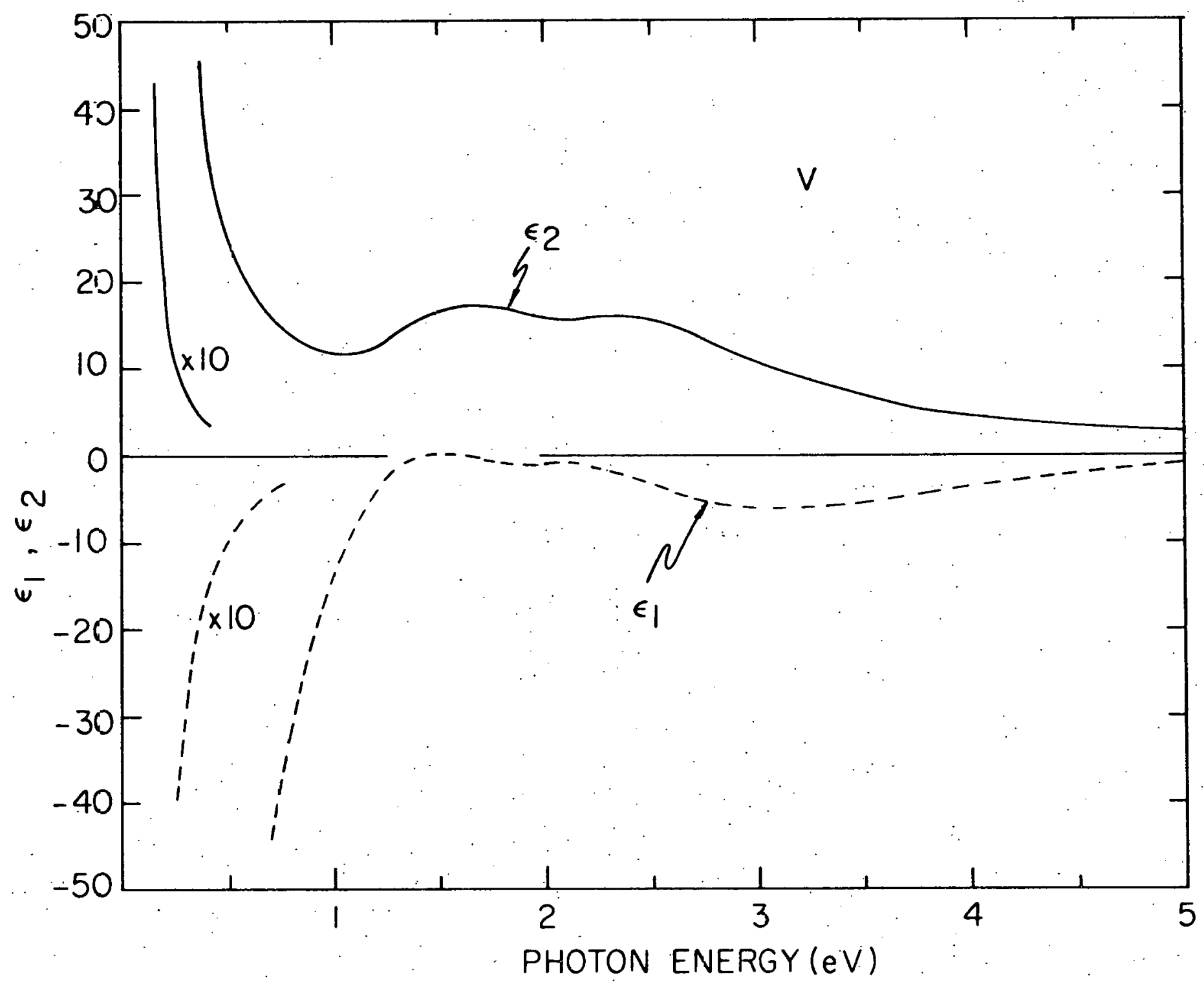

Figure 5. The real and imaginary parts of the dielectric function for $V$ 


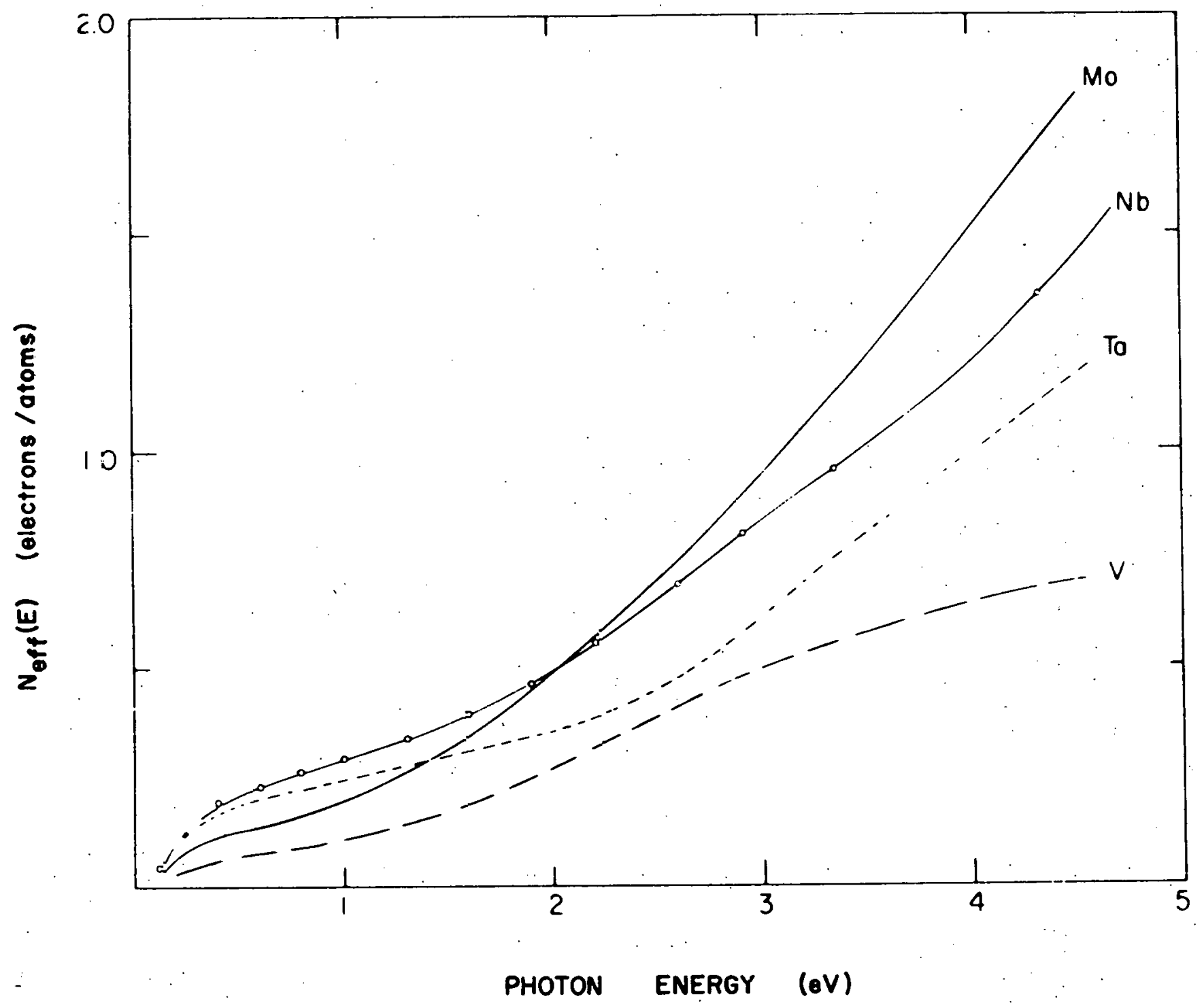

Figure 6. The calculated $\mathrm{N}_{\text {eff }}(\mathrm{E}) \mathrm{fcr} \mathrm{V}, \mathrm{Nb}, \mathrm{Ta}$, and Mo. The shapes of the $\mathrm{V}$ and Mo curves are reliable, but the magnitudes are probably not 
Niobium

The measured absorptivity of $\mathrm{Nb}$ is displayed in Figure 7 in the energy range 0.1 to $6 \mathrm{eV}$. The reflectivity above $5 \mathrm{eV}$ was determined by 01 son as discussed in Reference 81. Also shown are the results of Golovashkin, Leksina, Motulevich and Shubin (GLMS) (43) as calculated from their published $n$ and $k$ values. It should be noted that the lines associated with these latter data were drawn by the author and are not necessarily as GLMS might have drawn them. The data of Vilesov, Azgrubskii and Kirillova (VAK) (28) are also shown. It can be seen that the agreement of our ultraviolet data with those of VAK is reasonably good. Structures. In A at about 3.75 and $4.8 \mathrm{eV}$ are evident in both sets of data. The shoulder observed at lower energy at about $1.8 \mathrm{eV}$ is also apparent in the data of GLMS. Our data appear to be roughly $5 \%$ higher than those of GLMS and it is difficult to explain the difference. The results of GLMS and VAK clearly disagree at $3.1 \mathrm{eV}$, both in magnitude and slope, but this is the end of the experimental range of both groups. An examination of llie data polnts of the former shows the hint of a rise in $\mathrm{A}$ around $2.8 \mathrm{eV}$. If the data above $2.8 \mathrm{eV}$ were disregarded, an interpolated curve might easily be drawn to the data of VAK and the curve would run roughly parallel to ours.

An expanded low energy region allows a better comparison of our data with those of GLMS. In the insert of Figure 7, the three sets of data of GLMS as well as our own are shown. A comparison shows reasonable agreement of our. results with their $293 \mathrm{~K}$ data; they observe no structure in their room temperature results. However, the peaks at about 0.38 and 


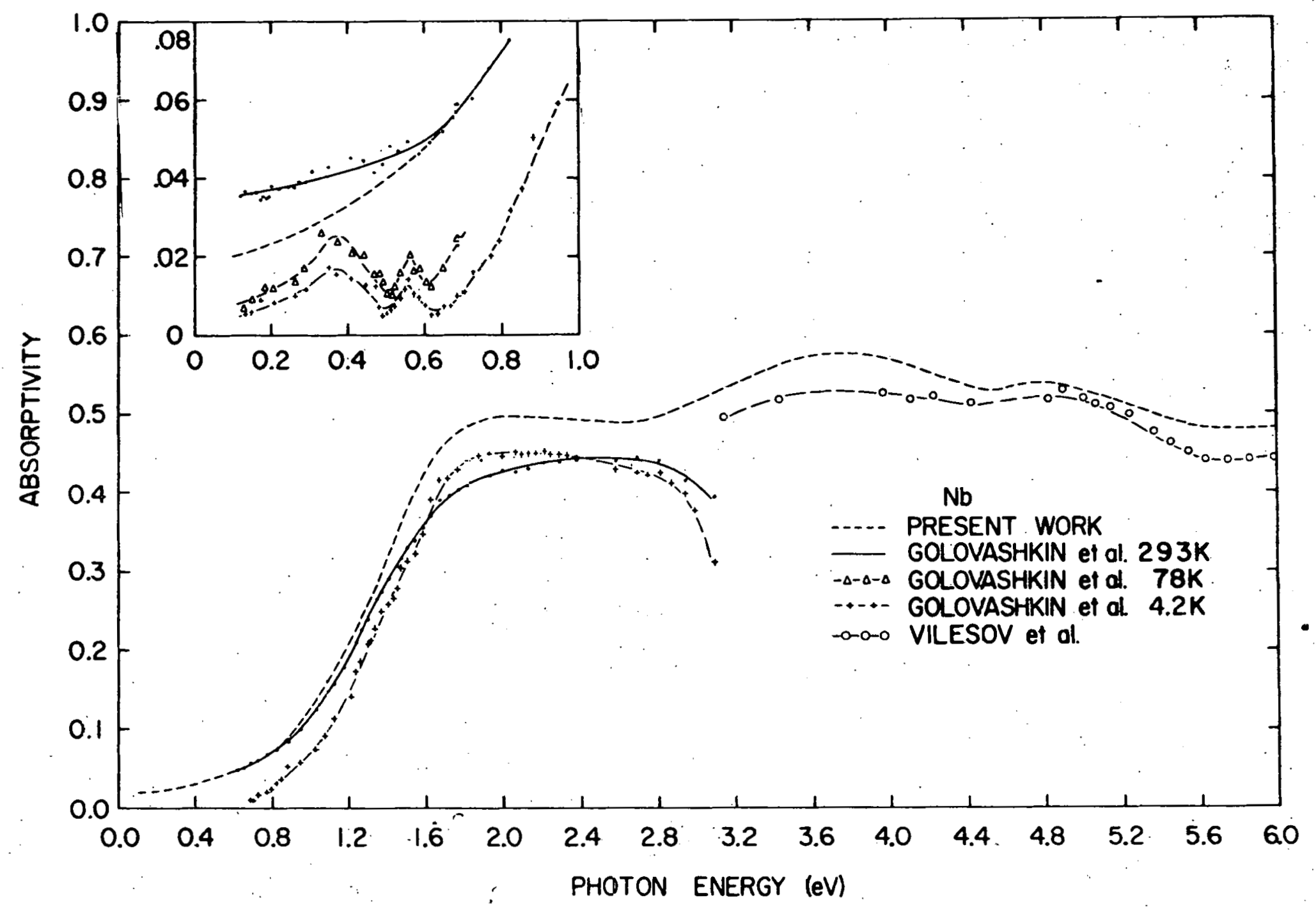

Figure 7. The absorptivity of $\mathrm{Nb}$ at $4.2 \mathrm{~K}$ anc near-normal incidence. The insert shows the low energy region 
$0.56 \mathrm{eV}$ which are seen in the low temperature work are anomalous, having appeared from nowhere with no counterpart in our spectrum. The existence of structure in $\mathrm{Zn}(19), \dot{C d}(18)$ and $A)(82)$ has been reported at 0.15 , 0.29 and $0.40 \mathrm{eV}$, respectively, and in $\mathrm{Ni}$ (83) slight changes of slope have been noted. These successes provide considerable confidence as to the rellabliticy and sensitivity of the calorimetric apparatus. From Figure 7, however, it is clear that no structure in $\mathrm{Nb}$ has been observed below the onset at about $\mathrm{l} \mathrm{eV}$. It is suspected that the results of. GLMS have been adversely affected by condensates on their samples at low temperature. It may be more than coincidence that $\mathrm{CO}_{2}$ and $\mathrm{H}_{2} \mathrm{O}$ possess strong absorption in the infrared between 1.4 and $7.5 \mu \mathrm{m}(0.9$ to $0.17 \mathrm{eV})$. Indeed at the energies where GLMS display peaks in $\mathrm{A}, \mathrm{CO}_{2}$ and $\mathrm{H}_{2} \mathrm{O}$ are essentially transparent but at energies on either side of the peaks; the spectra of the gases show strong absorption.

It might be argued that the disagreement arises from different sample preparation. Heeding the caution of GLMS regarding the removal of a damaged surface layer of approximately $50 \mu \mathrm{m}$ in depth, care was taken to remove amounts in excess of that amount. In an extreme case, a crystal was chemically reduced ( $\left.\mathrm{HF}: \mathrm{HNO}_{3}:: 1: 1\right)$ from 2.7 to $0.5 \mathrm{~mm}$ in thickness. The results obtained were the same as those presented in Figure 7, to within experimental error. Since it is known that an oxide layer forms very slowly on Nb any differences from that quarter can be disregarded, though certainly ellipsometry would be more sensitive to error than calorimetry.

In the Kramers-Kronig analysis, reflectivity data taken using the 
same sample (freshly cleaned, of course) were used to extend the range of $R(E)$ to $36.4 \mathrm{eV}$. At higher energies, extrapolations were introduced which took into account the presence of core transitions near $43 \mathrm{eV}$ (84, 85). The details of the experimental apparatus and data analysis for the high energy data have been given elsewhere $(81,86)$. We need note here only: that such data were available and were reliable. Beyond the range in the infrared, smooth extrapolations were used to take the absorptivity smoothly to zero.

In Figure 8 the real and imaginary parts of $\tilde{\epsilon}$ are shown. It is seen. that $\epsilon_{2}$ drops smoothly from a large, positive, free electron value in the infrared and passes through a minimum at 1.4 ev, thus marking the onset of dominance by interband rather than intraband effects. Subsequently, maxima are observed at $2.4,4.3$ and $5.1 \mathrm{eV} . \epsilon_{1}$ is seen to rise steeply, cross the axis at $1.95 \mathrm{eV}$ and peak at $2.2 \mathrm{eV}$. A maximum is then observed at $4.0 \mathrm{eV}$ with a minimum at $2.8 \mathrm{eV}$. The high energy results will not be discussed at this time.

The free electron plasma energy of $\mathrm{Nb}$ is calculated to be $19.56 \mathrm{eV}$, there being 5 electrons per atom, of which 1.42 have contributed to the sum rule between 0.1 and $4.5 \mathrm{eV}$. Neff is shown in Figure 6.

Tantalum

The refiectivity of Ta is shown in Figure 9. The results of 01 son are shown for energies above $5 \mathrm{eV}$; again, these need not concern us here. They have been discussed in detail elsewhere (87). The reflectivity falls smoothly from 0.98 at $0.15 \mathrm{eV}$ and reaches a minimum at $2.15 \mathrm{eV}(0.352)$ before climbing to a rather wide maximum at $3.4 \mathrm{ev}$. Another minimum has 


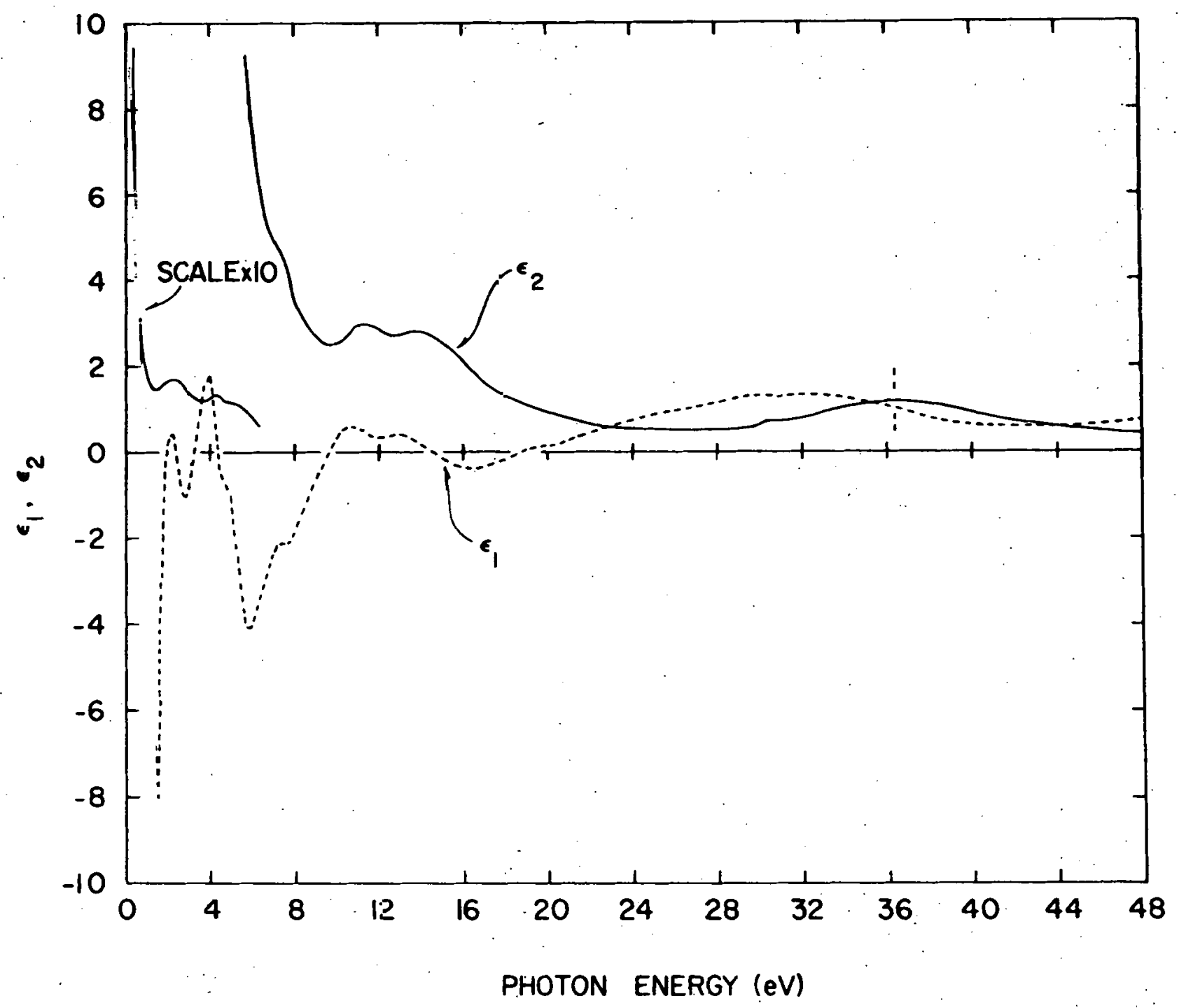

Figure 8. The dielectric constants =or $\mathrm{Nb}$ 


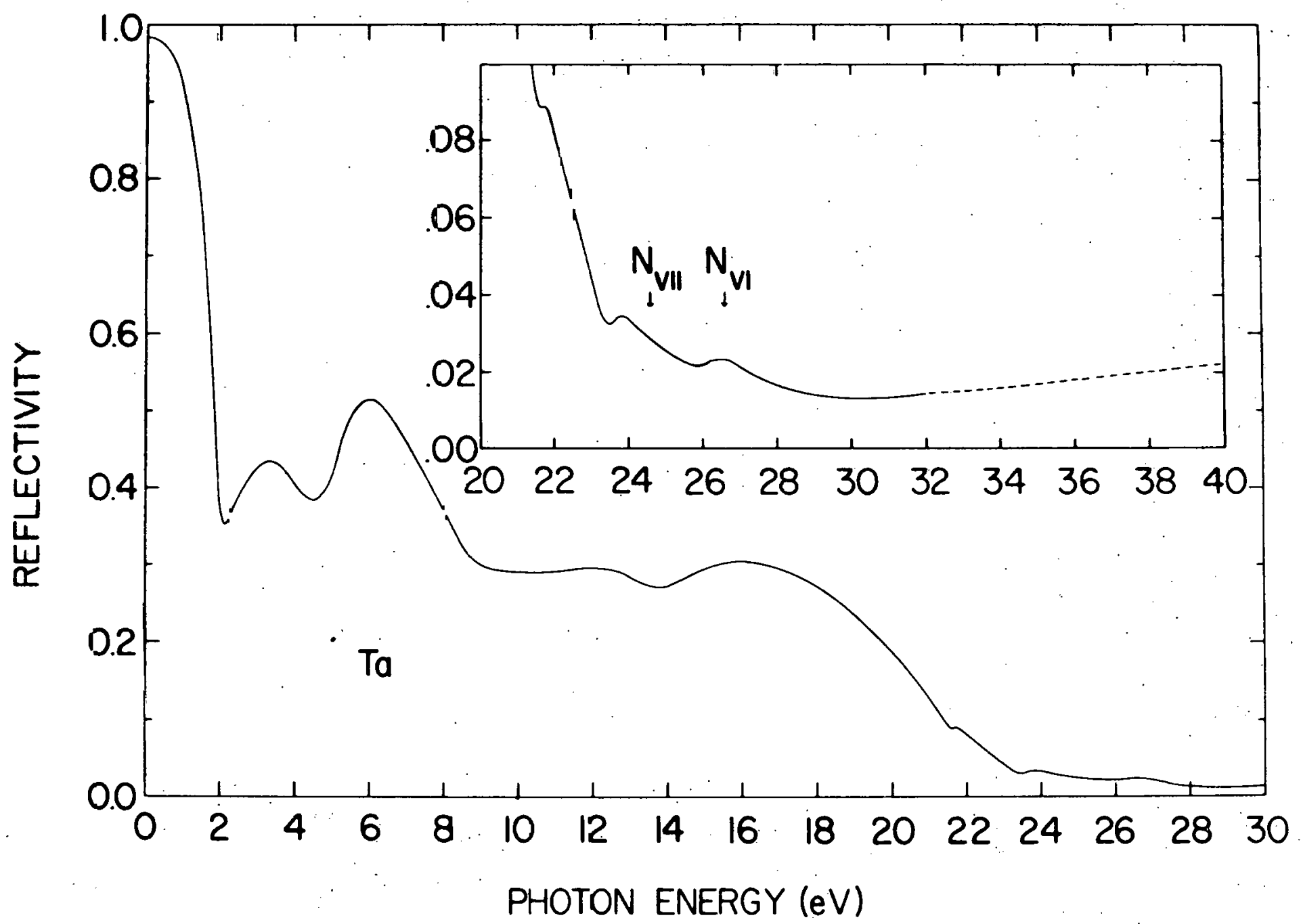

Figure 9. The reflectivity of Ta at near-nornal incidence. The data below 4.9 eV were obtained calorimetrically 
been observed at about $4.6 \mathrm{e} v$, followed by a large peak in the reflectivity at $6 \mathrm{eV}$. The agreement with the results of Juenker et al. $(50,51)$ in the visible and ultraviolet is rather satisfactory although at high energy, their $R$ values tend to be too large.

The availability of good high energy data made a KK analysis reliable. Thus, the reflectivity was continuous from 0.15 to $35 \mathrm{eV}$, above which $R(E)$ was calculated from the absorption measurements of Haensel et al. (88), which extended to $600 \mathrm{eV}$. Note that at $600 \mathrm{eV}$, $R$ was calculated to be only $0.0006 \%$ and it was legitimate to truncate the integral. Again, in the infrared an extrapolation was introduced to drop A to zero smoothly.

The real and imaginary parts of $\tilde{\epsilon}$ are shown in Figure $10 . \epsilon_{2}$ displays structure which can be identified as being interband in origin at 3.0 and $5.3 \mathrm{eV}$ while the dominant features in $\epsilon_{1}$ are maxima at 2.6 and $4.8 \mathrm{eV}$, and crossings of the axis at 2.0 and $5.3 \mathrm{eV}$. A calculation of $N_{\text {eff }}$ shows that 1.35 electrons per atom have contributed to the sum rule between 0.15 and $4.88 \mathrm{eV}$. The plasma energy was calculated to be 19.51, there being 5 -free elertrons per atom for Ta.

\section{Molybdenum}

The measured absorptivity of Mo is shown in Figure 11. No interband structure is evident in the infrared at energles less than about $1.5 \mathrm{eV}$ where the first maximum appears in $A$. The absorptivity rises smoothly from 0.016 at $0.15 \mathrm{eV}$. Following the peak at $1.5 \mathrm{eV}$, a second peak uccurs at about $2.2 \mathrm{eV}$, a minimum being at $1.85 \mathrm{eV}$. Finally, a broad shoulder appears to be centered at about $3.3 \mathrm{ev}$. Comparisons with the existing data on Mo shows that References 50-55 all show a maximum in $A$ 


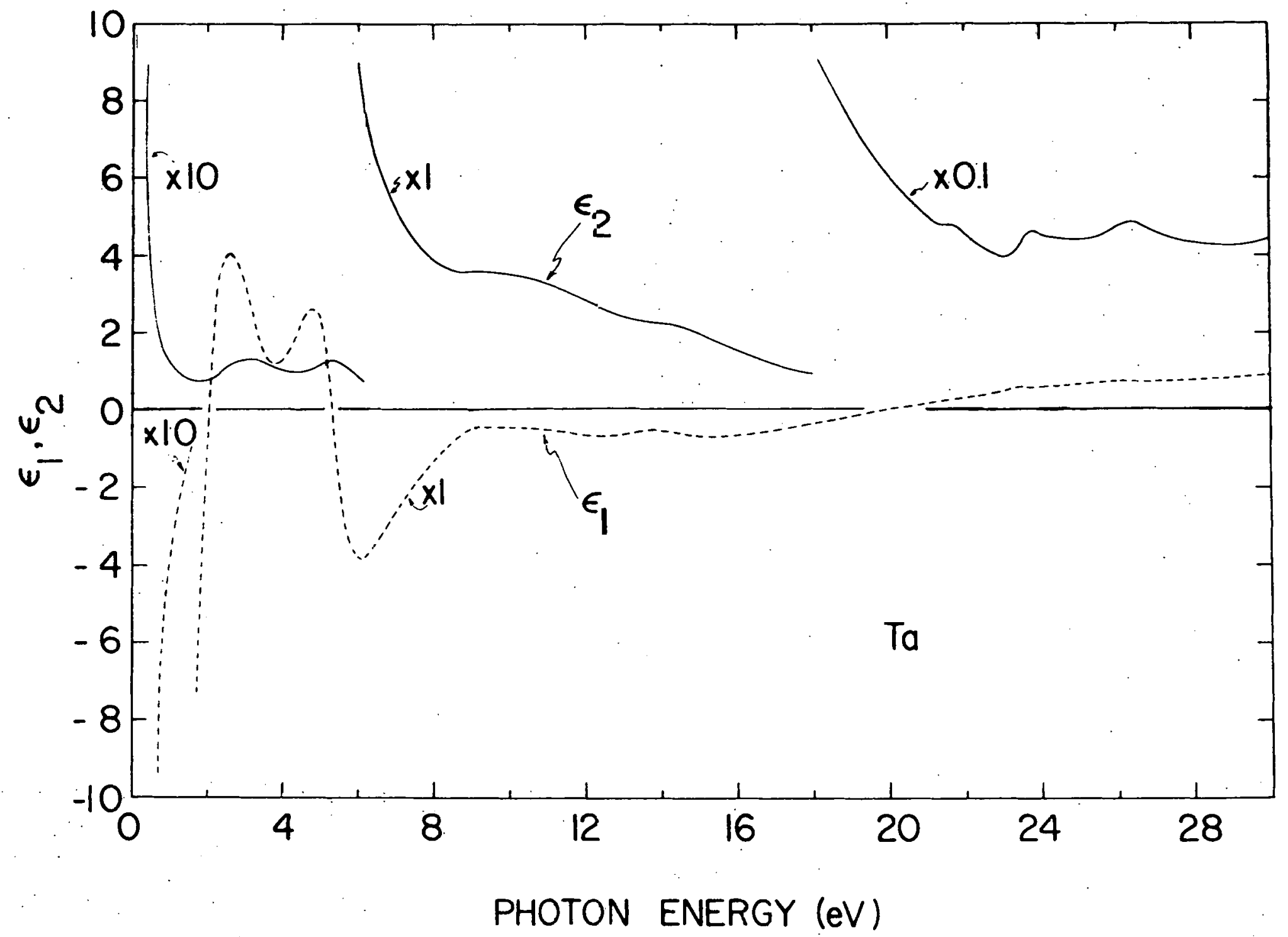

Figure 10. The dielectric constants for Ta 


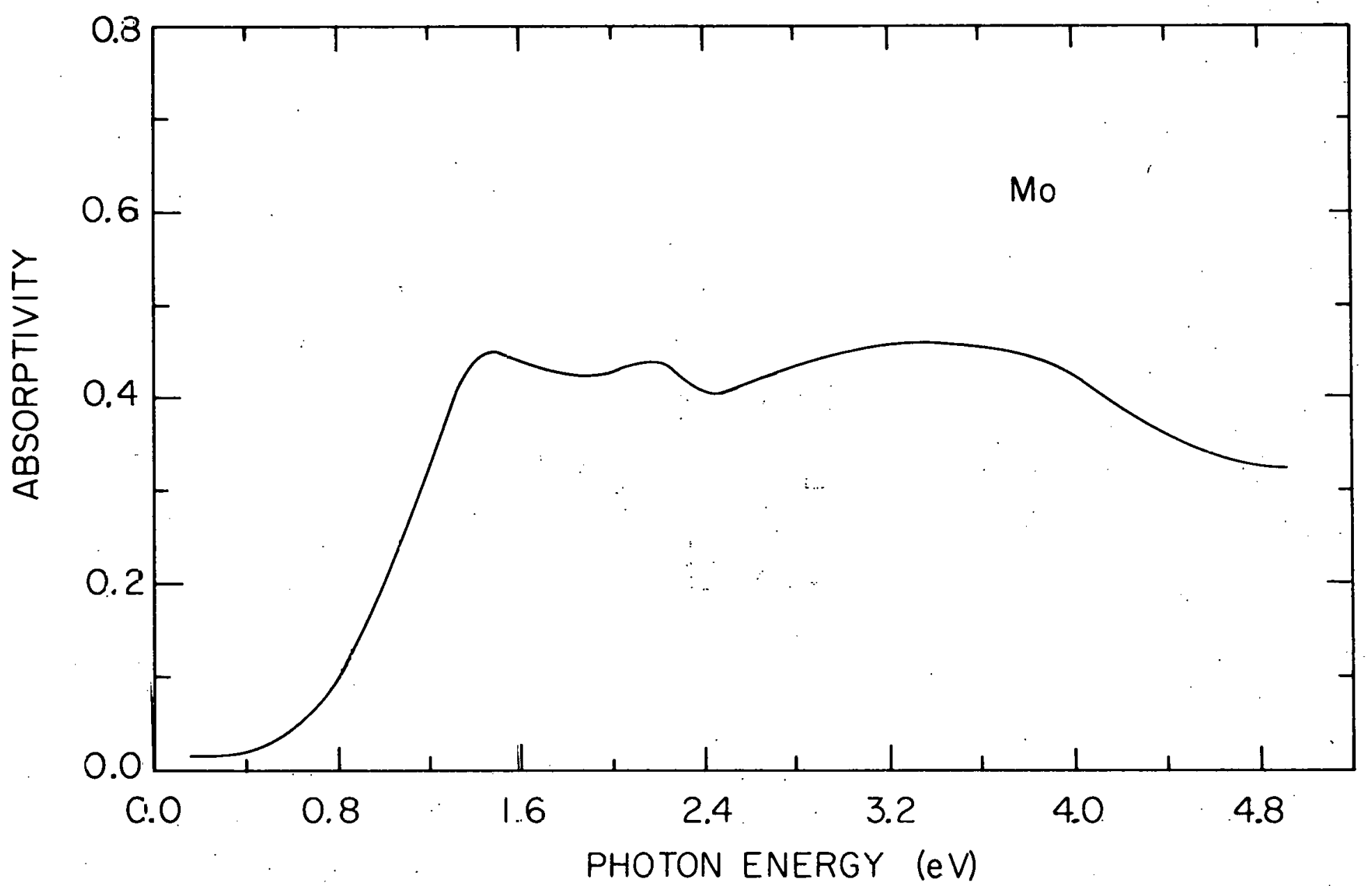

ज

Figure 11. The absorptivity of Mo at $4.2 \mathrm{~K}$ and near-normal incidence 
at about $3.5 \mathrm{eV}$, and those that extend into the infrared show a drop in A at about $1.3 \mathrm{eV}$. However, neither Reference 54 nor 55 shows a peak in $\mathrm{A}$ at $2.2 \mathrm{eV}$ and the minima at 1.8 and $2.5 \mathrm{eV}$ reported earlier by Kirillova et al. (52) and confirmed by us.

In the KK analysis of the data, it was necessary to rely upon the high energy data of Juenker et al. $(50,51)$. A comparison of their high energy reflectivity with those of Weaver et al. for Ta (87) has shown that their values are consistently high, suggesting a significant amount of scattered light. Accordingly, a scale factor was introduced to reduce the magnitude of $R$ above about $10 \mathrm{eV}$. It is thought that the effect on $\widetilde{\epsilon}$ below $5 \mathrm{eV}$ will be small. Nonetheless, the optical constants shown in Figure 12 are subject to change following the measurement of $R$ on our crystal in the vacuum ultraviolet.

The dielectric function is shown in Figure 12. Structure is apparent in $\epsilon_{2}$ at about $1.8,2.35$ and $4.1 \mathrm{eV} ; \epsilon_{1}$ crosses the axis at. 1.44 and $2.35 \mathrm{eV}$ and displays peaks at 1.6 and $2.25 \mathrm{eV}$ with a broad ridge centered at abnut $3,4 \mathrm{eV}$. The agreemenl with the results of Kirillova et al. (52) for Mo is quite good above about $1 \mathrm{eV}$, the values of $\epsilon_{1}$ and $\epsilon_{2}$ agreeing very well at l cV. Their values thus serve as a set of independently determined values and a check of the KK extrapolations and the reliability of lhe magnicudes shown in Figure 12. At lower energies, our absorptivity was lower than theirs and the dielectric function is modified arrondingly. The free electron plasma enerqy of Mo was calrulated to bc $23.03 \mathrm{eV}$. Between 0.15 and $4.88 \mathrm{eV}, 2$ of the 6 electrons free to participate in absorption have contributed to the sum rule. 


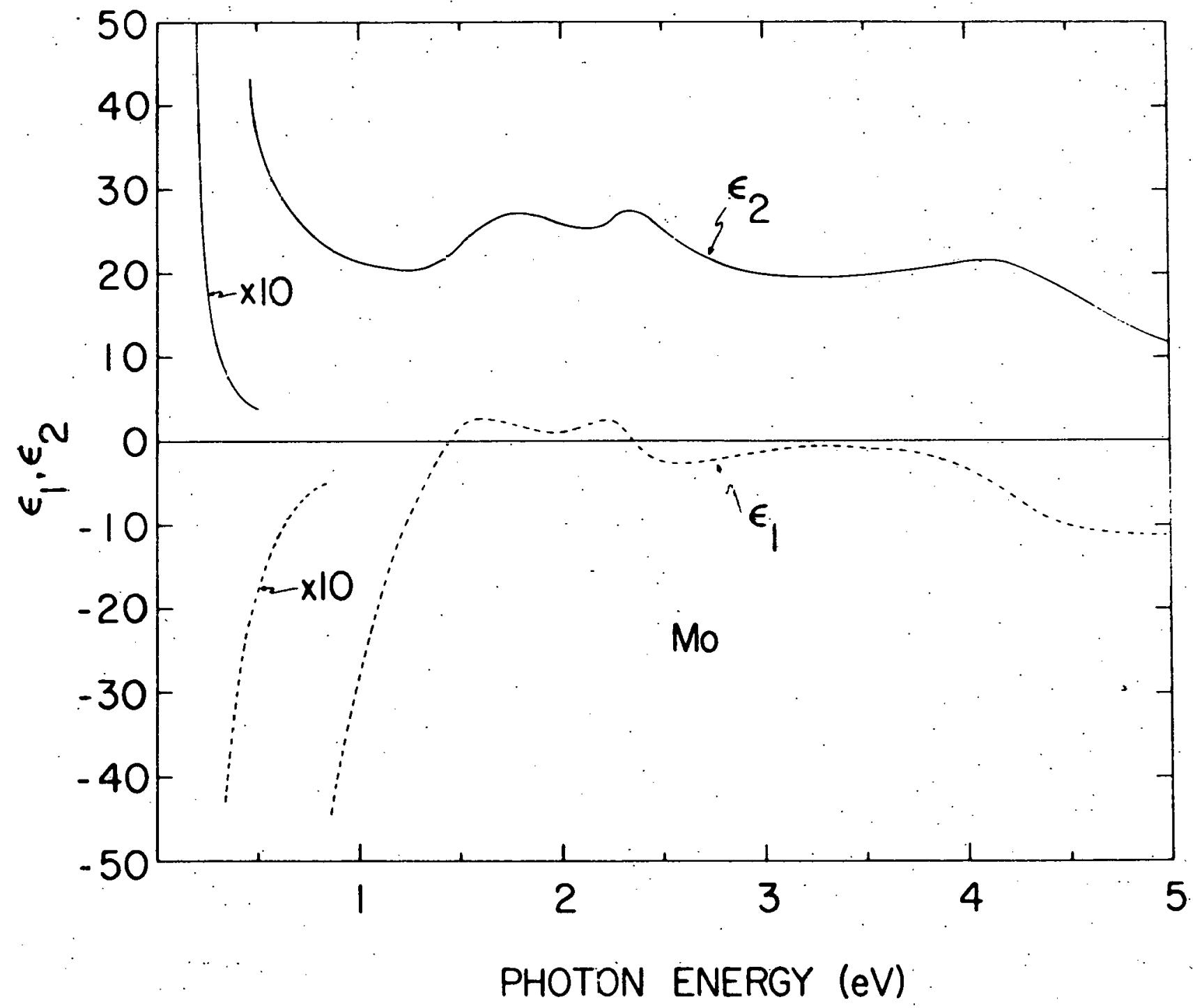

Figure 12. The dielectric constants for Mo 


\section{Yttrium}

The absorptivity spectra of $Y$ are shown in Figure 13. The anisotropy of $\vec{E} \| \hat{c}$ and $\vec{E}_{\perp} \hat{c}$ is quite pronounced be low about $\mathrm{I} \mathrm{eV}$; above about $1.5 \mathrm{eV}$, the spectra display similar structures with maxima occurring at 1.5 and 2.1 ev. Above about $2.6 \mathrm{eV}$, the spectra rise smoothly to the limit of the data $(4.42 \mathrm{eV})$. Of particular interest is the large infrared absorptivity. All of the bcc transition metals discussed above show a gradual rise of $A$ at low energy; none possess the precipitous rise in $A$ shown for $Y$. The magnitude of $A$ at $0.8 \mathrm{eV}$ ranges from 0.046 ( $\mathrm{Ta}$ ) to $0.134(V)$ while $Y$ has values of $0.373(\vec{E} \perp \hat{c})$ and $0.48(\vec{E} \| \hat{c})$. Further, while $Y$ displays a shouider in $A$ at $0.45 \mathrm{eV}(\vec{E} \| \hat{c})$, the other metals do not possess low energy structure below the first absorption peak.

As has been discussed above, no vuv data exist for $Y$, and, further, band calculations for $Y$ have not been performed'. Due to the absence of high energy data, a KK analysis has not been performed; to do so would be to artificially impose spectra and the optical constants could be widely inaccurate. Thelefure, the absorptivity spectra of $Y$ will be discussed in terms of the energy bands of Sc (13). While in general the reflectivity is a cumpllcated function of $\epsilon_{1}$ and $\epsilon_{2}$, it is hoped that at least qualitative information can be inferred about the electronic properties of $Y$.

1L. Hodges, lowa state Uliiversily. The band structure of $Y$ is presently being investigated. Private communication. 1972. 


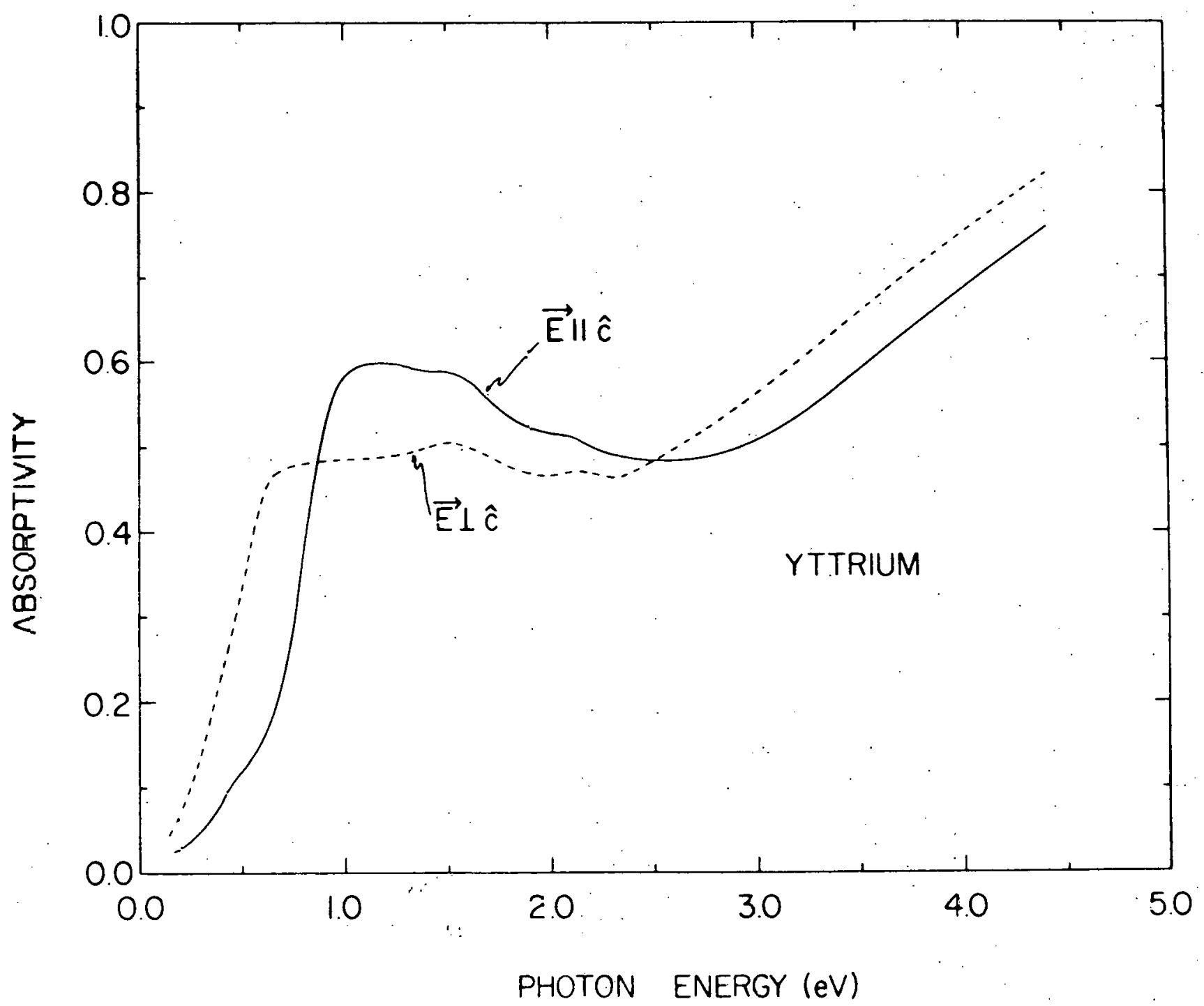

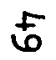

Figure 13. The absorptivity spectra for $Y$ at $4.2 \mathrm{~K}$, near-normal incidence 


\section{DISCUSSION}

The general procedure for interpreting the structure in the dielectric function is to compare observed structure with energy band calculations, if they exist, or to propose a model which will explain the experimental results if such bands have not been calculated. This is generally based on the bands of similar metals; for example, $Y$ and Sc are expected to have similar band schemes and the bands of Sc could be modified to explain structure in $Y$.

At low energies, free carrier absorption usually dominates interband effects. As in Equation, 8, if $\omega \ll \omega_{p}$ and $\omega \tau \gg>1$, the absorptivity can be written $A=2 / \omega_{p} c$, which is independent of energy and holds only for a free electron gas with the electronic density of a metal. The anomalous skin effect theory has shown this expression to be inadequate, but it will suffice for the moment. Then, deviations from a flat absorption curve might be attributed to interband effects, but the assumptions that $\omega \ll \omega_{p}$ and $\omega \tau \gg 1$ must be examined carefully before drawing such rnnclusions, since, as one begins to violate those conditions, the absorptivity becomes energy dependent.

For the transition metals under discussion, the plasma energies, dc resistivities (89), relaxation times (at $4.2 \mathrm{~K}$ ), values of $\omega \tau$ (at $4.2 \mathrm{~K}$ ), and predicted, free electron absorption values are given in Table 3. From the table It is evident that for $\mathrm{V}, \mathrm{Nb}$ and Mo the contribution to $\mathrm{A}$ from the free electron gas is small. For Ta and $Y$, the contributions are larger and the condltion on wt is not clearly met. While this is true, even for Ta and $Y$ the contribution from free carriers is small relative 
to the measured absorptivities at low energy. Further, though A may not be a constant, the rate at which it is increasing is not sufficient to match the measured rate. It appears, then, that interband effects do contribute significantly even at low energy.

Table 3. Free electron characteristics of the samples

\begin{tabular}{|c|c|c|c|c|c|}
\hline Sample & $\begin{array}{l}\text { Plasma } \\
\text { Energy } \\
(e v)\end{array}$ & $\begin{array}{l}\text { Resist Ivity } \\
(295 \mathrm{~K}) \\
(\mu \Omega-\mathrm{cm})\end{array}$ & $\begin{array}{l}\text { Relaxation } \\
\text { Time at } 4.2 \mathrm{~K} \\
\left(10^{-16} \mathrm{sec}^{-1}\right)\end{array}$ & $\mathrm{k}(4.2 \mathrm{~K})$ & $\begin{array}{c}\text { Free Carrier } \\
\text { Absorption }\end{array}$ \\
\hline v & 22.30 & 19.9 & 4.95 & 22.5 & 0.00060 \\
\hline $\mathrm{Nb}$. & 19.56 & 14.5 & 8.85 & 20.1 & 0.00076 \\
\hline$T a$ & 19.51 & 13.1 & 9.83 & 4.85 & 0.00317 \\
\hline Mo & 23.03 & 5.33 & 17.33 & 76.1 & 0.00017 \\
\hline$Y \| \hat{c}$ & 11.19 & 34.6 & 11.32 & 6.15 & 0.00450 \\
\hline$\gamma \perp \hat{c}$ & 11.19 & 71.6 & 5.47 & 3.52 & 0.00760 \\
\hline
\end{tabular}

The Interband dielectric function $\varepsilon_{2}$ was shown in Equation 10 to contaln a factor termed the joint density of states (JDOS). The JDOS is large where two bands are nearly parallel and are separated by the Fermi level, as seen by the behavior of the denominator of the JDOS. Structure in $\varepsilon_{2}$ is then interpreted in terms of such bands, but subject to a condition on the matrix elements of Equation 11. As usual, selection rules exist which allow or forbid transitions from the initial state to the final state. Since the possible symmetries of the wave functions are 
determined by the symmetry of the crystal lattice, group theory can be applled and the selection rules calculated. Hence, structure in $\varepsilon_{2}$ can be related to allowed transitions, the strength being large where the Joos is large.

Structure will occur in $\varepsilon_{2}$ where the denominator of Equation 11 vanishes. Such points are termed critical points and will occur, for example, at points of high symetry where both bands are flat. Different types of critical points exist, and, in the case of semiconductors, it is worthwhlle to denumerate and study them, each giving rise to a characteristlc shape of $\varepsilon_{2}$. However, for metals this does not seem worthwhle since interpretation based on critical points has not been very successful. Instead of sharp structure in $\varepsilon_{2}$, metals typically. possess broad features which make critical point identiflcations very difficult. The commonest critical point phenomena in metals is the general critical point, where the denominator in Equation 11 vanishes but nelther term in the denominator vanishes. Thus there are two parallel bands of fliled and empty states, of ten occupying considerable regions of $\vec{k}$-space.

The energy bands are typically calculated at points of high symmetry and along llnes of symmetry in the Brillouin zone. The correlation between theory and experiment is then limited to a discussion of these points, the bands at points of arbitrary $\vec{k}$ vector not being known. Accordingly, when one might determine a range of energles in which transitions between two bands might be expected, structure in the measured $\varepsilon_{2}$ is not sharp and limited to that range. Rather; since 
contributions to $\varepsilon_{2}$ are arising from nearby regions of $\vec{k}$-space, as well as the polnts and lines in question, the features are typically broad and smooth. When tentative identifications of the origin of structure in $\varepsilon_{2}$ is made in subsequent sections, this should be borne in mind.

\section{Vanadium}

Self-consistent band calculations for $V$ have been performed by Yasui et al. (37) and Papaconstantopoulos et al. (36); the latter group earlier reported their results for nonself-consistent calculations (35). Less detailed calculations have also been carried out by Snow and Waber (33) and by Mattheiss (32). The investigations of Yasui et al. employed two different coefficients of the exchange potential, $\lambda=1$ and 0.725 , and the resulting bands were quite different. In particular, in their calculation, the position of $\Gamma_{25}^{\prime}$ was quite dependent on the choice of $\lambda$. For $\lambda=1$, the position was below the Fermi level while for $\lambda=0.725$ it lay above, in agreement with the results of all the other calculations mentioned. Accordingly, these bands are preferred and are reproduced in Figure 14 .

While the band calculations show considerable agreement as to the overall shape of the bands, important differences exist. The Yasui et al. results (Figure 14) show $\Delta_{5}$ touching the Fermi level; the results of Papaconstantopoulos et al. show $\Delta_{5}$ well above the level, as do the preliminary results of Hodges (38). Likewise, Yasui et al. display the $\Sigma_{1}$ band dipping well into the Fermi surface while Papaconstantopoulos et al. show it not touching $(\lambda=1)$, and barely contacting $(\lambda=0.67)$.

The width of the $d-b$ and is also a matter of considerable interest and 


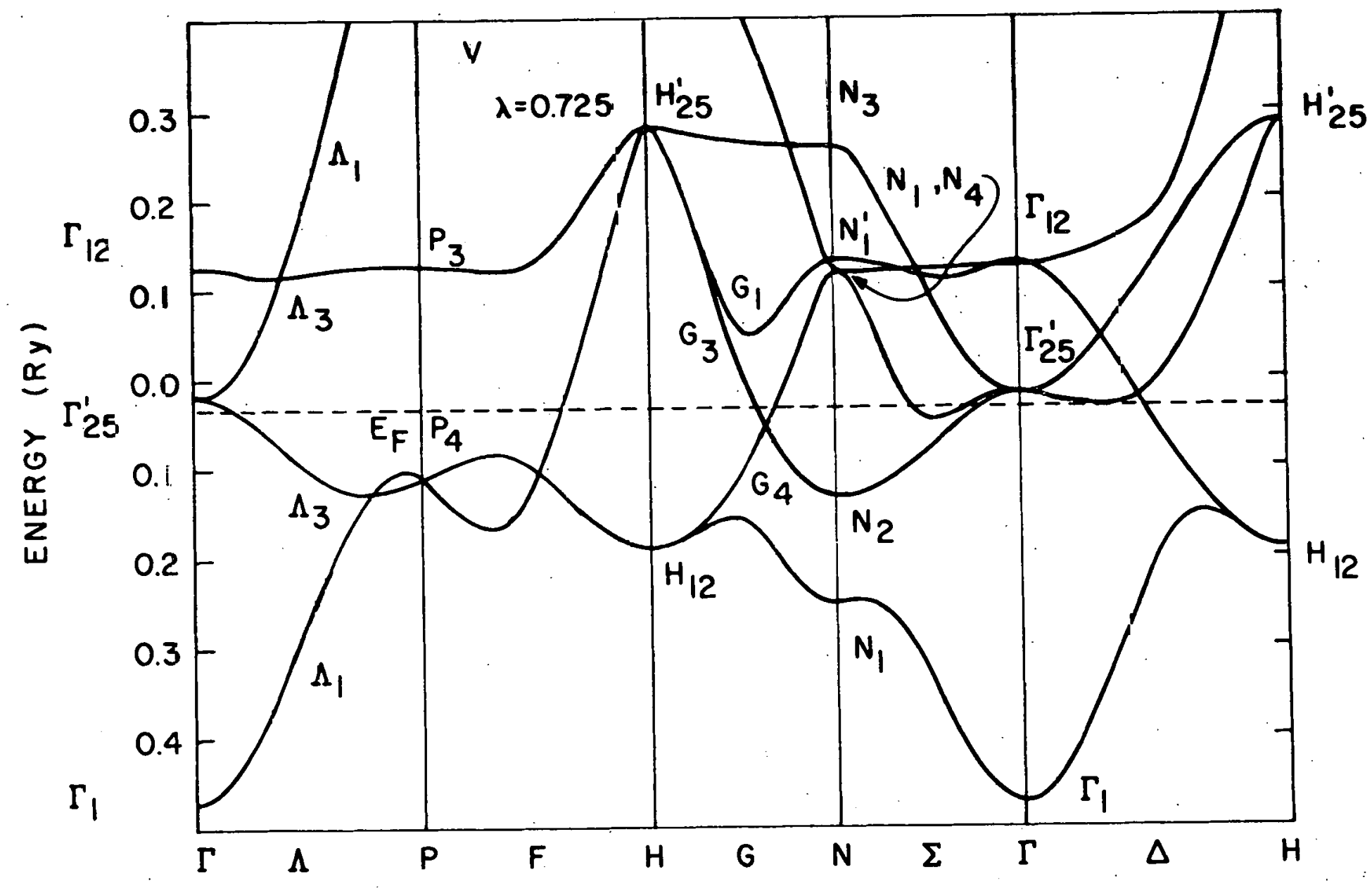

Figure 14. Energy bands of V caiculated by Yasui et al. (Reference 37) 
disagreement... If it is defined in the usual sense for a bcc crystal, $E\left(\mathrm{H}_{25}{ }^{\mathrm{i}}\right)-\mathrm{E}\left(\mathrm{H}_{12}\right)$, then a comparison of the different calculations gives: Mattheiss (32) $6.5 \mathrm{eV}$; Hodges et al. (14) $7.9 \mathrm{eV}$; Yasui et al. $6.45 \mathrm{eV}$ $(\lambda=0.725)$ and $5.4 \mathrm{eV}(\lambda=1)$; Anderson et al. (35) (nonself-consistent) $6.8 \mathrm{eV}(\lambda=1)$ and $11 \mathrm{eV}(\lambda=0.67)$; Papaconstantopoulos (self-consistent) $5.5 \mathrm{eV}(\lambda \equiv 1)$ and $1.27 \mathrm{eV}(\lambda=0.67)$. With these differences in mind, we proceed to discuss the electronic properties of $V$ with the hope of indicating a preference amont the various bands:

In. Figure 4 the absorptivity of $V$ was shown and it was shown that there was no structure in A below about $1.35 \mathrm{eV}$; rather, the absorptivity rises smoothly. From figure 5, we see that the behavior of $\varepsilon_{2}$ is basically free-electron like below $0.8 \mathrm{eV}$. Above about $1 \mathrm{eV}$, interband effects are clearly entering in as seen by the minimum in $\varepsilon_{2}$. Structure then appears at 1.65 and $2.45 \mathrm{eV}$ although sharp maxima are not observed. From the bands of Figure 14, it is possible to identify tentatively the origin of the $1.65 \mathrm{eV}$ structure as transitions near $G, G_{3}$ and $G_{4}$ are seen to cross the Fermi level and are separated from $G_{1}$ by roughly $1-1.5 \mathrm{eV}$. This feature of the $V$ bands is also seen in the bands of Papaconstantopoulos et al. although the energy separation is closer to $2 \mathrm{eV}$. Likewlse, from the two sets of bands, the band which is shown to dip below (or contact) the Fermi level near $\Sigma$ is also a prime candidate for the structure. The estimated energy difference ranges from 1 to $1.8 \mathrm{eV}$. This latter transition will subsequently be identified as the origin of low energy structure in $\mathrm{Nb}$ and $\mathrm{Ta}$ and is the favored identification. Corroborating evidence for either transition comes from the thermomodulation results of weaver 
et al. (42) in which structure in $\Delta \varepsilon_{2}$ was reported at $1.35 \mathrm{eV}$. The magnltude of the structure suggests $E_{F} \rightarrow$ unoccupied band, as these both are. As was pointed out earller, the bands have been calculated only along symmetry lines and the structure is probably supplemented by nearby regions of $\vec{k}$-space.

Structure in $\varepsilon_{2}$ was also observed at $2.45 \mathrm{eV}$. In the thermomodulation results, the signal at about $2.45 \mathrm{eV}$ was much smaller than that at $1.35 \mathrm{eV}$, suggesting that the fermi level is not involved in the transitions. The bands near $P$ are separated by $2.5-3 \mathrm{eV}$ and are possibly the origin of the rather broad structure. The bands of Papaconstantopoulos et al. disagree as to the magnitude of the separation (about $4 \mathrm{eV}$ ), but provide possible alternatives. The bands from $P$ to $N$ (not shown in Figure 14) are separated by energies of about $2-2.5 \mathrm{eV}$.

The identifications made here must be considered quite tentative. The results of Yasul et al. appear to be unsatisfactory in certain parts of the Brillouin zone (notably in the directions $\Gamma(\Sigma) N$ and $\Gamma(\Delta) H$ ) and may well be in error elsewhere. Their agreement with the de Haas-van Alphen data (41) has not been discussed, but comparison with the photoemission results of Eastman ( 31 ) shows that their calculated occupled $d$ width $\left(E_{F}-H_{12}\right)$ is only $2.16 \mathrm{eV}$ compared to the measured value of $3 \mathrm{eV}$. The agreement of the Papaconstantopoulos et al. results is better $(3.28 \mathrm{eV})$ but, as they polnted out, the de Haas-van Alphen agreement is only qualitative ( $15 \%$ smaller than experiment). The agreement would be improved, they reported, by increasing the value of $\lambda$. Since an increase in $\lambda$ tends to broaden the d-band, the comparison with Eastman's results would suffer. 
Niobium

APW band calculations have been carrled out by Mattheiss (6) for Nb and $\mathrm{Ta}$, the latter. including relativistic effects. A comparison of the Nb bands with those of Deegan and Twose (5) shows that the overall agreement is quite satisfactory. Agreement is also quite good with the results of Anderson et al. (49). The recent application of the empirical pseudopotential method to $\mathrm{Nb}$ has proved to be successful and again Mat theiss' bands were the standard.

In Figure 7 the absorptivity spectrum was displayed for the low energy range, and it was concluded above that interband effects do not produce stiructure below the $1.0 \mathrm{eV}$ onset. The band scheme shows that indeed the only transitions (of those calculated) which could be identified as those observed by GLMS are $\Sigma_{2} \rightarrow \Sigma_{1}$. For a bcc crystal, these are forbidden by dipole selection rules. The effect of spin-orbit splitting, however, is to cause these bands to become $\Sigma_{5}$ bands and the corresponding $\Sigma_{5}+\Sigma_{5}$ transitions are allowed. However, since spin-orbit effects are not large in $\mathrm{Nb}$, such transitions are thought to be weak.

Structure was observed in $\varepsilon_{2}$ at $2.4 \mathrm{eV}$ (see figure 8). While the transitions $\Sigma_{2}+\Sigma_{1}$ are forbidden, $\Sigma_{1} \rightarrow \Sigma_{1}$ are allowed. A tentative identification of the structure is then $\Sigma_{1} \rightarrow \Sigma_{1}$, and nearby regions of $\vec{k}$-space. The density of states for the bands involved is large but the JDOS is not known. These bands correspond to those discussed in $V$ and give rlse to seructure which appears at higher energy than in $v$.

Weaker interband transitions can also be expected from the $\Gamma_{1}+\Gamma_{25}^{\prime}$ pair at about $5.7 \mathrm{eV}$ and $\Delta_{1}+\Delta_{2}^{\prime}$ at about $4.4 \mathrm{eV}$. These might account 


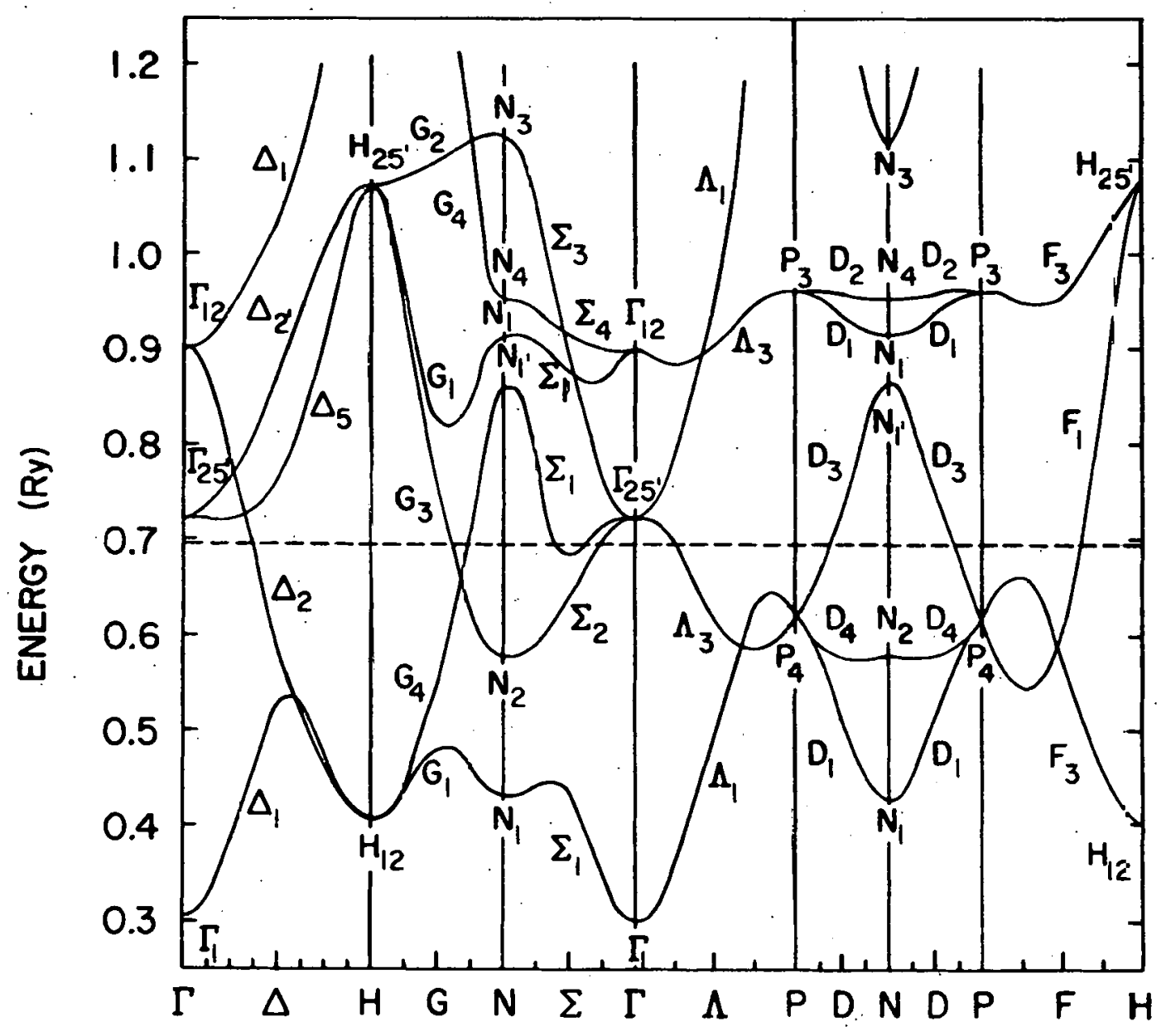

Figure 15. Energy bands for Nb as icalculated by Mattheiss (Reference 6) 
for the experimental structure observed at about those energies. Transitions between the bands along $P(D) N$ would also contribute at about $5 \mathrm{eV}$ $\left(\mathrm{N}_{2}-\mathrm{N}_{4}\right.$ is about $\left.5.2 \mathrm{eV}\right)$ and might better account for the $5.3 \mathrm{eV}$ structure. Weaker transitions from $\left(\Gamma_{1}-\Lambda_{1}\right) \rightarrow\left(\Gamma_{25}^{\prime}-\Lambda_{1}\right)$ could also be entering into the picture; the bands are only roughly parallel and their joint density of states would be accordingly reduced.

\section{Tantalum}

The bands of Ta were calculated by Mattheiss when he considered those of $\mathrm{Nb}$. A comparison of the two fermi surfaces was made by him and by Halloran et al. (46). Considerable similarity was found, as expected. Comparison of the reflectivity results of figure 9 with those of Juenker et al. $(50,51)$ shows good agreement insofar as the position of structure is concerned. The agreement in the magnitudes is less satisfactory. Between 2 and $5 \mathrm{eV}$, our results have more clearly defined structure; comparison of the higher energy data shows that their $R$ magnitudes are considerably higher than ours, perhaps due to scattered light in their apparatus.

A comparison of the reflectivities of $\mathrm{Nb}$ and $\mathrm{Ta}$ shows that $\mathrm{Ta}$ has the less complicated spectrum. Between 1 and $10 \mathrm{eV}$, minima (maxima) occur in $\mathrm{Nb}$ at $2.0,3.8,4.8$ and $7.3 \mathrm{eV}(2.6,4.5,5.8$ and $7.7 \mathrm{eV})$ while for Ta minima (maxima) were observed at 2.15 and $4.6 \mathrm{eV}(3.4$ and $6 \mathrm{eV}$ ). Thus, both metals have the first strong absorption peak near $2 \mathrm{eV}$ and share the common structure at $6 \mathrm{eV}$. The dissimilarity of the other structures is difficult to correlate accurately to energy bands, but clearly the bands and densities of states are less.similar. away from the 
Fermi level than the Fermi surface measurements would indicate.

In the infrared, the absorptivities of both metals rise smoothly without structure below $2 \mathrm{eV}$. In the case of $\mathrm{Nb}$ it was argued that transitions like $\Sigma_{2} \rightarrow \Sigma_{1}$ were forbidden by selection rules but that spinorbit splitting would remove that restriction. For Nb the spin-orbit splitting was thought to be small, but in Ta it might be expected to be larger, Ta being a heavier metal. Accordingly, the spectral region was carefully searched for structure in $A$; none was observed. Presumably, such transitions, if they exist, are very weak. As the state of the art of low temperature, infrared derivative spectroscopy is improved, perhaps structure will yet be revealed. In particular, it would show up in the rmomodulation experiments since the fermi level is involved in at least some of the transitions.

Tentative identifications of the structure observed in the dielectric function can be made as based on the Ta bands of Figure 16. The structure in $\varepsilon_{2}$ at $3.0 \mathrm{eV}$ can be compared with the $2.4 \mathrm{eV}$ structure in $\mathrm{Nb}$ and the $1.65 \mathrm{eV}$ structure in V. All of these presumably arise from $\Sigma_{1} \rightarrow \Sigma_{1}$ transitions. The calculated energy separation of the bands is increasing with the progression from $3 d$ to $4 d$ to $5 d$ metal, as observed here. Transitions like the $\Gamma_{1} \rightarrow \Gamma_{25}^{\prime}$ transitions in $\mathrm{Nb}$ which occurred at about $5.7 \mathrm{eV}$ have been shifted to about $8 \mathrm{eV}$ for Ta. Since no structure was observed at about $8 \mathrm{eV}$ in Ta, it is qulte likely that such transitions are very weak in both metals. Structure is observed at $5.4 \mathrm{eV}$ and the bands near $\mathrm{N}$ are separated by about $5 \mathrm{eV}$; these may be the origin of that structure. A comparison with the features of $\mathrm{Nb}$ shows that structure also appears at 


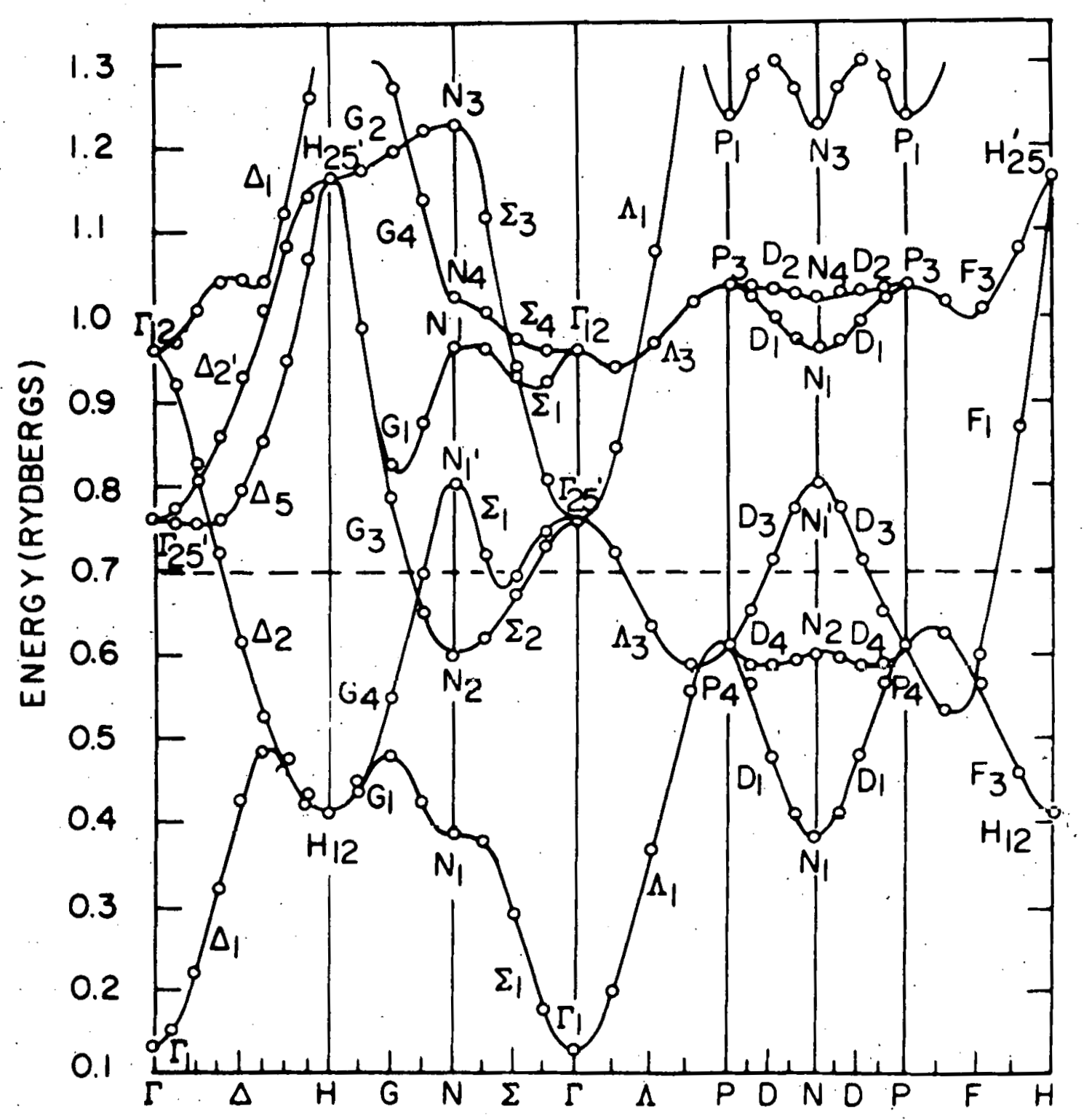

Figure 16. Energy bands for Ta by Mattieiss (Reference 6) 
about $5 \mathrm{eV}$ and the same transitions (near $N$ ) could be responsible. It is probable, then, that the common feature arises from those transitions near $N$ for both $\mathrm{Nb}$ and $\mathrm{Ta}$.

\section{Molybdenum}

The bands calculated by Iverson and Hodges (73) are shown in Figure 17. The spin-orbit coupling parameter was estimated to be only one-fourth. of that for $W(0.00744$ vs. 0.0275 Ry) and the section of the band which only slightly dips below the Fermi level for $W$ is known to provide the well-known lenses of Mo. The effects of spin-orbit coupling on the bands near the Intersection of the electron jack and the lens (along $\Gamma(\Delta) H$ ) have been shown by Mattheiss (72) and Loucks (70) for $W$. The effects are less pronounced for Mo but the bands hybridize near their crossing and separate, leaving their average energy unchanged. The degeneracy in the bands along $\Delta_{5}$ and $\Delta_{2}^{\prime}$ is also lifted. These effects have not been'ishown In Figure 17, but it is clear that vertical transitions from the lower to the upper band along $\Delta$ would give rise to structure in $\varepsilon_{2}$ at about $1-2 \mathrm{eV}$. Accordingly, the observed $\varepsilon_{2}$ structure at $1.8 \mathrm{eV}$ can be identified as orlginating from such transitions, those and from nearby regions of $\vec{k}$-space. It should be noted that while $\varepsilon_{2}$ displays a smooth rise above. about $1.2 \mathrm{eV}$, the lowest value of $\varepsilon_{2}$ is about 20 , which indicates that interband effects are already important at $1.2 \mathrm{eV}$. Thls has already been discussed with reference to the low energy behavior of $A$.

An identification of the structure in $\varepsilon_{2}$ at 2.35 and $4.1 \mathrm{eV}$ must be cautious since, presumably some of the bands of interest have been split by spin-orbit effects and the magnitude of the splitting is not completely 


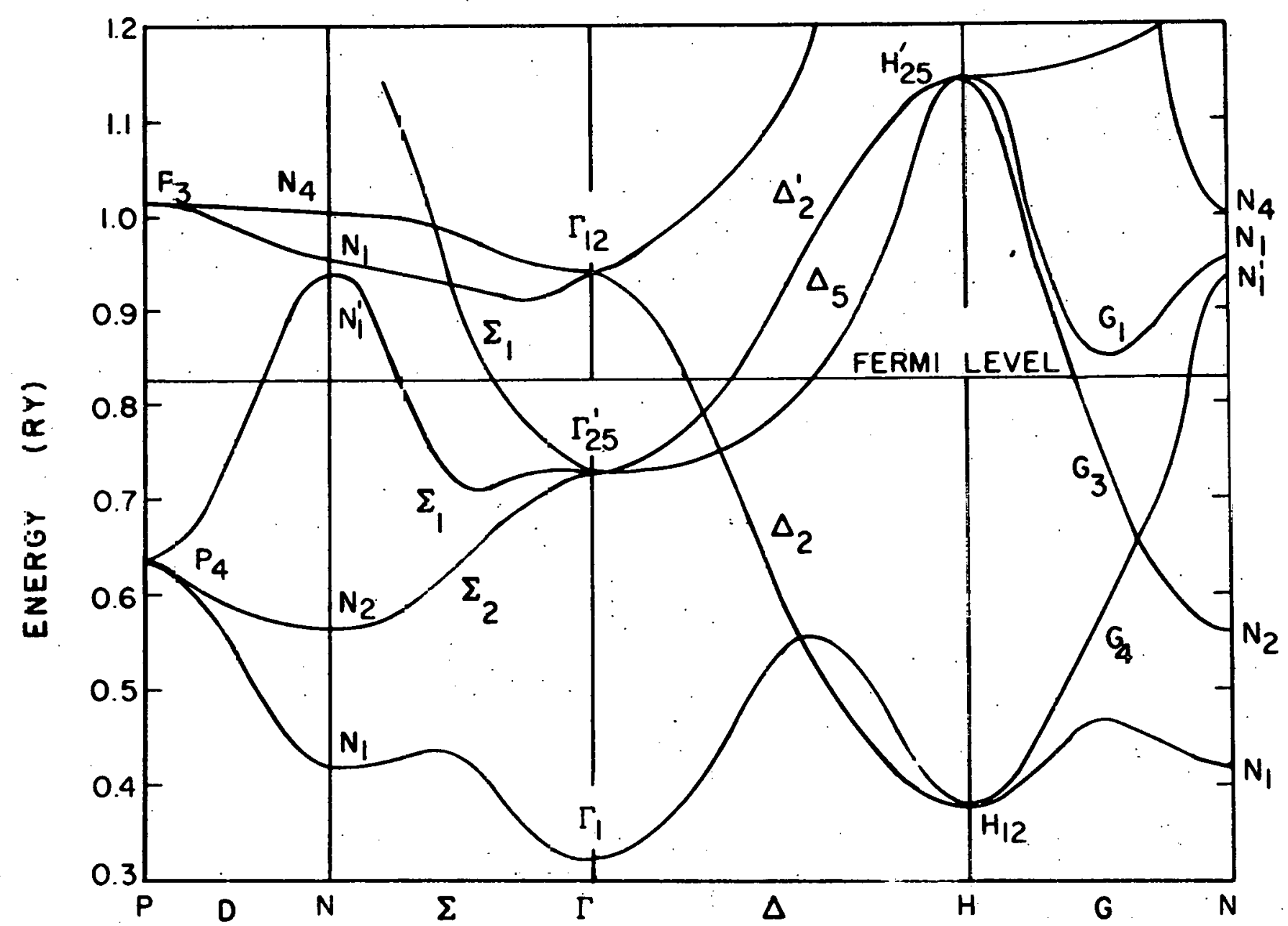

a

Figure 17. Energy bands for Mo by verson and Hodges (Reference 73). Spin-örbit effects are not shown 
known (though it is thought to be small). Thus, for example, transitions from $\Gamma_{25}$, to $\Gamma_{12}$ may be affected by the lifting of the degeneracy at $\Gamma_{25^{\circ}}^{\prime}$. With this in mind, we suggest that transitions like $\Gamma_{25}^{\prime} \rightarrow \Gamma_{12}$ and $\Sigma_{1} \rightarrow \Sigma_{1}$ and nearby regions of $\vec{k}$-space contribute to the structure at $2.35 \mathrm{eV}$ and transitions like those from $\Sigma_{1} \rightarrow \Sigma_{3}$ might account for the structure at $4.1 \mathrm{eV}$.

\section{Yttrium}

As discussed earlier, there have been no complete band calculations for $Y$, but there is considerable similarity between $Y$ and SC and the SC bands should allow tentative interpretation of the $Y$ experimental results. The bands for Sc are shown in Figure 18 and are due to Fleming and Loucks (13).

From the absorptivity shown in Figure 13, a slight shoulder can be seen at about $0.45 \mathrm{eV}$ for $\vec{E} \| \hat{c}$. For Sc, parallel bands cross the Fermi surface along $T$ and are separated by about $0.25 \mathrm{eV}$. For Gd (78) and Re (77), the same bands are less parallel but still are cut by the Fermi level and are separated by low energies. These $T_{1}$ and $T_{2}$ bands probably persist in $Y$ and would give rise to the observed anisotropy in the low energy absorptivity curves. The transitions are allowed for $\vec{E} \| \hat{c}$ but forbidden by selection rules for $\vec{E}_{+} \hat{c}(90)$. Further, transitions like those from $\Sigma_{3}$ to $\Sigma_{1}$. are allowed for $\vec{E} \| \hat{c}$ and appear in Sc separated by about $0.6 \mathrm{eV}$; since the bands are not parallel, the joos is small and the structure would be relatively weak. Transitions at $H$ and nearby reglons of $\vec{k}$-space would likewise contribute to the structure of Figure 13 . For 


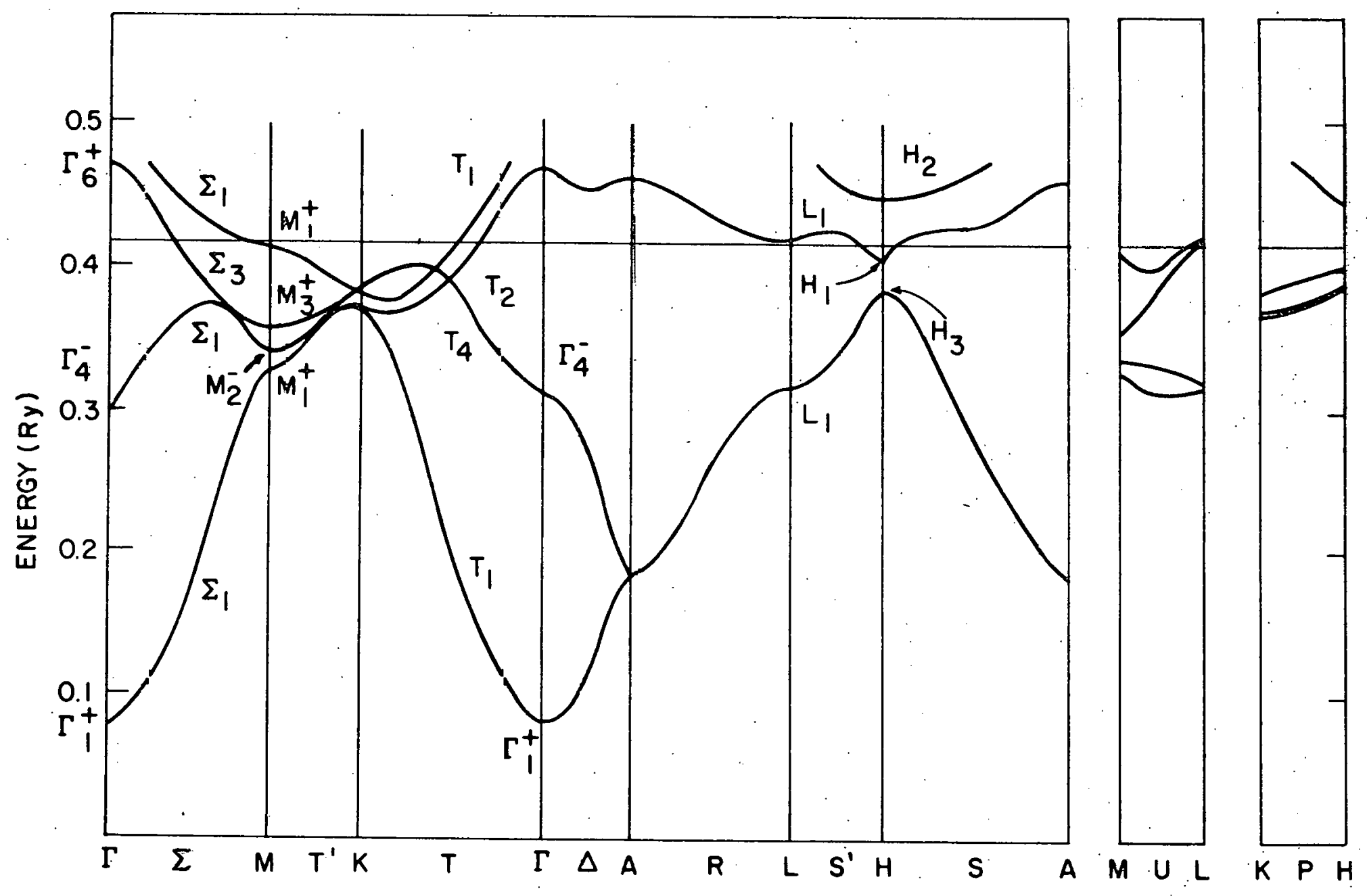

Figure 18. Energy bands of Sc by Fleming and Loucks (Reference 13) 
Sc, such transitions as $\mathrm{H}_{3} \rightarrow \mathrm{H}_{2}\left(0.9 \mathrm{eV}\right.$, both polarizations) and $\mathrm{H}_{1} \rightarrow \mathrm{H}_{2}$ ( $\approx 0.5 \mathrm{eV}, \vec{E} \perp \hat{c}$ only) transitions are apparent and would probably persist in $Y$. Finally, transitions like $\mathrm{L}_{l} \rightarrow \mathrm{L}_{\mathrm{I}}(1.4 \mathrm{eV}$ in Sc, not calculated for Gd and suppressed more deeply before $E_{F}$ for Re) might account for further absorption. It is quite impossible to make even qualitative estimates of the origins of the higher energy structures. 


\section{CONCLUSIONS}

A comparison of the spectra of the series $V B$ bcc metals $V, N b$, and Ta shows that there is considerable similarity in the low energy region. All possess a first strong absorption peak between 1 and 2.5 $\mathrm{eV}$ (1.35 eV for $\mathrm{V}$, about $2 \mathrm{eV}$ for $\mathrm{Nb}$ and $2.15 \mathrm{eV}$ for Ta). It was argued above that the origin of the corresponding structure in $\varepsilon_{2}$ is probably from transitions like $\Sigma_{1} \rightarrow \Sigma_{1}$. The observed shift of the experimental structure to higher energy as one proceeds down the VB series has been theoretically predicted for $\mathrm{Nb}$ and $\mathrm{Ta}$ as shown by the respective bands. While there is some uncertainty concerning the behavior of the lower band in $V$, it is suggested that it does contact the Fermi level, as shown by Papaconstantopoulos et al.

The bands connecting $P$ to $N$ were shown to be reasonably parallel for $\mathrm{Ta}$ and $\mathrm{Nb}$ with energy separations $\left(\mathrm{N}_{4}-\mathrm{N}_{2}\right)$ of about $5.0 \mathrm{eV}(\mathrm{Nb})$ and $5.7 \mathrm{eV}$ (Ta), with large densities of states associated with them. Yasui et al. (37) did not consider the direction $P(D) N$, but did show. the separation $\mathrm{N}_{4}-\mathrm{N}_{2}$ to be about $3.7 \mathrm{eV}$. Likewise Papaconstantopoulos et al. (36) also predicted about $3.8 \mathrm{eV}$ separation. The structures in $\varepsilon_{2}$ for $\mathrm{Nb}(5.3 \mathrm{eV})$ and $\mathrm{Ta}(5.4 \mathrm{eV})$ were attributed to these transitions. In V, however, structure appears at lower energy $(2.45 \mathrm{eV})$. Tentative identifications of the origin of the structure based on the bands. of Figure 14 have been made above and it is not likely that the bands. along $P(D) N$ are involved. Papaconstantopoulos et al. suggested that their choice of $\lambda$ was too small, but an increase would probably 
increase the d-band separation and the gap would increase. This would be in better agreement with the preliminary results of Hodges (38) which showed $\mathrm{N}_{4}-\mathrm{N}_{2}$ to be about $4.7 \mathrm{eV}$. It is quite possible that when our reflectivity results become available for $V$ at higher energies, structure wi 11 be observed between 5 and $6 \mathrm{eV}$ that was not noted by Seignac and Robin (29). This would introduce structure in $\varepsilon_{2}$ at about the same energy as observed in $\mathrm{Nb}$ and $\mathrm{Ta}$. This is quite speculative, but the high energy reflectivity results and the completed bands of Hodges should be quite informative. Certainly, preliminary comparisonf of the $\checkmark$ bands of Hodges with those of Mattheiss (16) ( $T a$ and $\mathrm{Nb}$ ) show better behavioral consistency than similar comparison of the Mattheiss results with those of Yasui et al. or Papaconstantopoulos et al.

Thus, the series VB metals have been considered in some detail. With the high energy reflectivity results for Mo and $V$, the dielectric constants can be calculated reliably and the optical properties of the hcc transition metals $\mathrm{V}$, Nh, Ta and Mn should be complete between 0.15 and $36 \mathrm{eV}$. While $Y$ cannot be studied in the vuv, the results in the visible and infrared should prove useful in band calculations of $Y$. 


\section{BI 8LI OGRAPHY}

1. B. Segall, Phys. Rev. 125, 109 (1962).

2. H. Ehrenreich and H. R. Philipp, Phys. Rev. 128, 1622 (1962).

3. T. L. Loucks, Augmented Plane Wave Method (Benjamin; New York, 1967).

4. J. M. Ziman, Solid State Physics Vol. 26 (Academic Press, New York, 1970)?

5. R. A. Deegan and W. D. Twose, Phys. Rev. 164, 993 (1967).

6. L. F. Mattheiss, Phys. Rev. B1, 373 (1970).

7. W. A. Harrison, Phys. Rev. 181, 1636 (1969).

8. W. A. Harrison, Pseudopotentials in the Theory of Metals (Benjamin, New York, 1966).

9. C. Y. Fong and M. L. Cohen, Phys. Letters 40A, 482 (1972). .'

10. S. L. Altmann and C. J. Bradley, Phys. Rev. 135, A1253 (1964).

11. S. L. Altmann and C. J. Bradiey, Proc. Phys. Soc. (London) 22 , 704 (1967).

12. T. L. Loucks, Phys. Rev. 159, 544 (1967).

13. G. S. Fleming and T. L. Loucks, Phys. Rev. 173, 685 (1968).

14. L. Hodges, R. E. Watson and H. Ehrenreich, Phys. Rev. B10, 3953 (1972).

15. H. Ehrenreich and M. L. Cohen, Phys. Rev. 115, 786 (1959).

16. M. Cardona, Solid State Physics Vol. Il (Academic Press, New York, 1969).

17. R. V. Kasowski, Phys, Rev. 187, 885 (1969).

18. R. J. Bartlett, D. W. Lynch and R. Rósei, Phys. Rev. B3, 4074 (1971).

19. J. H. Weaver, D. W. Lynch and R. Rosel, Phys. Rev. 85, 2829 (1972).

20. F. Stern, Solid State Physics 15, 300 (1963). 
21. J. S. Toll, Phys. Rev. 104, 1760 (1956).

22. H. E. Bennett and J. M. Bennett, Physics of Thin Films Vol. 4, ed. by G. Hass and R. E. Thun (Academic Press, New York, 1967).

23. E. Passaglin, R. R. Stromberg and J. Kruger, eds., Ellipsometry in the Measurement of Surfaces and Thin Films (National Bureau of Standards Misc. Publications 256, 1964).

24. M: Ph. Stoll, J. Appl. Phys. 42,1717 (1971).

25. M. M. Kirillova, Soviet Phys. JETP $\underline{34}, 178$ (1972).

26. F. A. Schmidt, lowa State University, private communication, 1972.

27. G. A. Bolotin, A. N. Voloshinskii, M. M. Kirillova, M. M. Noskov, A. V. Sokolov and B. A. Charikov, Fiz. Metal. Metalloved. 13, 823 (1962).

28. F. I: Vilesov, A. A. Azgrubskii and M. M. Kirillova, Optika i Spektros. 23, 153 (1967).

29. A. Seignac and S. Robin, C. R. Acad. Sci. Series B 271, 919 (1970).

30. B. Sonntag, R. Haensel and C. Kunz, Solid State Comm. Z, 597 (1969):

31. D. Eastman, Solid State Comm. 7, 1697 (1969).

32. L. F. Mattheiss, Phys. Rev. 134, A970 (1964).

33. E. C. Snow and J. T. Waber, Acta Metallurgica 17, 623 (1969).

34. L. F. Mattheiss, Phys. Rev. 139, Al893 (1965).

35. J. R. Anderson, J. W. McCaffrey and D. A. Papaconstantopoulos, Solid State Comm. Z, 1439 (1969).

36. D. A. Papaconstantopoulos, J. R. Anderson and J. W. McCaffrey, Phys. Rev. 85, 1214 (1972).

37. M. Yasui, E. Hagashi and M. Shimiza, J. Phys. Soc. Japan 29, 1446 (1970).

38. L. Hodges, lowa State University, private communication, 1.972.

39. N. E. Alekseevski $i$ and V. S. Egorov, Soviet Phys. JETP Letters 1 , 141 (1965). 
40. K. S. Nelson, J. L. Stanford and F. A. Schmidt, Phys. Letters 28A, 402 (1968).

41. R. A. Phillips, Phys. Letters 36A, 361 (1971).

42. J. W. Weaver, C. Culp and D. W. Lynch, to be published.

43. A. I. Golovashkin, T. E. Leksina, G. P. Motulevich and A. A. Shubin, Soviet Phys. JETP 29, 27 (1969).

44. M. M. Kirillova and B. A. Charikov, Fiz. Metal. Metalloved. 19, 495 (1965).

45. E. Fawcett, W. A. Reed and R. R. Soder, Phys. Rev. 159, 533 (1967).

46. M. M. Halloran, J. H. Condon, J. E. Graebner, J. E. Kunzler and F. S. L. Hsu, Phys. Rev. Bl, 336 (1970).

47. A. C. Thorson and T. G. Berlincourt, Phys. Rev. Letters Z, 244 (1971):

48. G. B. Scott, M. Springford and J. R. Stockton, Phys. Letters $27 A$, 655 (1961).

49. J. R. Anderson, J. W. McCarthey and D. A. Papaconst antopoulos, Bull. Am. Phys. Soc. 16, 637 (1971).

50. D. W. Juenker, L. J. LeBl anc and C. R. Martin, J. Opt. Soc. Am. 58, 164 (1968).

51. L. J. LeBlanc, J. S. Farrell and D. W. Juenker, J. Opt. Soc. Am. 54, $456(1964)$.

52. M. M. Kirillova, L. V. Nomerovannaya and M. M. Noskov, Soviet Phys. JETP 33; 1210 (1971).

53. M. M. Kirillova; G. A. Bolotin and V. M. Mayesvskii, Fiz. Metal. Metalloved. 19, 13 (1965).

54. M. L. Kapitsa, Yu. P. Udoev and E. I. Shirokikh, Soviet Phys. Solid State 11, 665 (1969).

55. K. A, Kress and G. J. Lapeyre, J. Opt, Soc. Am. 60, 1681 (1970).

56. L. V. Nomerovannaya, M. M. Kirillova and M. M. Noskov, Soviet Phys. JETP $\underline{33}, 405$ (1971).

57. J. A. Hoekstra and J. L. Stanford, Phys. Rev., to be published. 
58. D. M. Sparklin and J. A. Marcus, Phys. Rev. 144, 484 (1965).

59. G. Leaver and A. Myers, Phil. Mag. 19, 465 (1969).

60. V. R. Bolko, V. A. Gasparov and 1. G. Gverdtsiteli, Soviet Phys. JETP 29, 267 (1969).

61. J. R. Cleveland and J. L. Stanford, Phys. Rev. B4, 311 (1971).

62. E. Fawcett, Phys. Rev. 128, 154 (1962).

63. E. Fawcett and W. A. Reed, Phys. Rev. 134, A723 (1964).

64. N. E. Alekseevski i, V. S. Egorov. G. E. Karstens and R. N. Kazak, Soviet Phys. JETP 15, 519 (1963).

65. E. Fawcett and D. Griffiths, J. Phys. Chem. Solids 23, 1631 (1962).

66. P. A. Bezuglyi, S. E. Zhevago and V. T. Denisenko, Soviet Phys. JETP 22, 1002 (1966).

67. R. Herrman, Phys. Stat. Sol. 25 , 661 (1968).

68. W. M. Lomer, Proc. Phys. Soc. (London) 80, 489 (1962).

69. W. M. Lomer, Proc. Phys. Soc. (London) 84, 327 (1964):

70. T. L. Loucks, Phys. Rev. 139, Al181 (1965).

71. L. Hodges, lowa State University, private communication, 1972.

72. L. F. Mattheiss, Phys. Rev. 139, A1893 (1965).

73. R. J. Iverson and L. Hodges, to be submitted to Phys. Rev.

74. J. P. Petrakian, J. P. Palmari and G. Rasigni, Appl. Optics 9, $2115(1970)$ :

75. T. L. Loucks, Phys. Rev. 144, 504 (1966).

76. T. L. Loucks, Phys. Rev. 159, 544 (1967).

77. L. F. Mattheiss, Phys. Rev. 143, 506 (1966).

78. J. 0. Dimmock and A. J. Freeman, Phys. Letters 13, 750 (1964).

79. S. C. Keeton, Ph.D. thesis; lowa State University 1966 (unpublished). 
80. L. Bos, Ph.D. thesis, lowa State University, 1969 (unpublished).

81.. J. H. Weaver, D. W. Lynch and C. G. Olson, Phys. Rev. accepted for publication.

82. L. W. Bos and D. W. Lynch, Phys. Rev. B2, 4784 (1970).

83. D. W: Lynch, R. Rosei and J. H. Weaver, Solid State Comm. 9, 2195 (1971).

84. H. R. Apholte and K. Ulmer, Phys. Letters 22, 552 (1966).

85. M. J. Lynch and J. B. Swan, Aust. J. Phys. 21, 811 (1968).

86. D. W. Lynch and C. G. Olson, to be published.

87. J. H. Weaver, D. W. Lynch and C. G. 01 son, to be published.

88. R. Haensel, K. Radler, B. Sonntag and C. Kunz, Solid State Comm. Z, 1495 (1969).

89. G. T. Meaden, Electrical Resistance of Metals (Plenum; New York, 1965).

90. J. F. Cornwell, Phys. Kondens. Materie 4, 327 (1966). 


\section{ACKNOWLEDGMENTS}

A great many people have contributed to this work and to the various projects with which the author was involved. These contributions have ranged from concrete measurements to theoretical assistance to helpful suggestions and technical assistance. As is usually the case, this work has been carried out against a background of semi-continuous banter and kibitzing. To my comrades who have contributed this, their friendship and encouragements; I say thanks, it's been great!

It has been a pleasure to be associated with Professor David $W$. Lynch. His guidance and assistarice are gratefully appreciated.

The collaboration with Dr. C. G. Olson and Mr. C. Culp has been most productive; their contributions cannot be minimized. Thanks also to Dr. M. A. Lind for measuring the absorptivity of Mo to $20^{\circ} \mu \mathrm{m}$.

Special thanks also go to F. A. Schmidt for providing the crystals used in this study; to H. H. Baker for assistance with electropolishing and crystal handling; to A. D. Johnson for annealing the crystals; to G. V. Austin, E. L. Dekalb and N. M. Neymer for the chemical analysis of the materials; to J. 0 . Oslensun for determining the resistivity ratios.

Discussions with Professor L. Hodges have been most helpful and are greatly appreciated.

It is with great appreciation that I thank my wife, Carol, for her patlence, understanding and support during the years of graduate study. 